\title{
Tropical Cyclone Track Sensitivity in Deformation Steering Flow
}

\author{
RyAN D. TORN, TRAVIS J. Elless, AND PhILIPPE P. PAPIN ${ }^{\mathrm{a}}$ \\ Department of Atmospheric and Environmental Sciences, University at Albany, State University of New York, Albany, New York \\ CHRISTOPHER A. DAVIS \\ National Center for Atmospheric Research, Boulder, Colorado
}

(Manuscript received 29 April 2018, in final form 19 July 2018)

\begin{abstract}
Previous studies have suggested that tropical cyclones (TCs) in deformation steering flows can be associated with large position errors and uncertainty. The goal of this study is to evaluate the sensitivity of position forecasts for three TCs within deformation wind fields [Debby (2012), Joaquin (2015), and Lionrock (2016)] using the ensemble-based sensitivity technique applied to European Centre for Medium-Range Weather Forecasts (ECMWF) ensemble forecasts. In all three cases, the position forecasts are sensitive to uncertainty in the steering wind within $500 \mathrm{~km}$ of the 0 -h TC position. Subsequently, the TC moves onto either side of the axis of contraction due to the ensemble perturbation steering flow. As a TC moves away from the saddle point, the ensemble members subsequently experience different ensemble-mean steering winds, which act to move the TC away from the ensemble-mean TC position along the axis of dilatation. By contrast, the position forecasts appear to exhibit less sensitivity to the steering wind more than $500 \mathrm{~km}$ from the initial TC position, even though the TC may interact with these features later in the forecast. Furthermore, forecasts initialized at later times are characterized by significantly lower position errors and uncertainty once it becomes clear on which side of the axis of contraction the TC will move. These results suggest that TCs in deformation steering flow could be inherently unpredictable and may benefit from densely sampling the near-storm steering flow and TC structure early in their lifetimes.
\end{abstract}

\section{Introduction}

One of the great achievements of numerical weather prediction (NWP) has been the significant reduction in tropical cyclone (TC) track errors. This improvement is often attributed to improved model resolution, physics, and data assimilation systems (Rappaport et al. 2009). With that being said, there is evidence that the improvement in 0-72-h track forecasts is beginning to level off (e.g., Landsea and Cangialosi 2018); therefore, further reductions in track error may have to be achieved by addressing cases that are characterized by large track errors relative to the mean value (e.g., Yamaguchi et al. 2017). In many of these situations, the track forecast is quite sensitive to specific features, such as upper-tropospheric troughs, leading to anisotropic position variability (i.e., position variability that

\footnotetext{
${ }^{a}$ Current affiliation: Naval Research Laboratory, Monterey, California.
}

Corresponding author: Ryan Torn, rtorn@albany.edu occurs preferentially in one coordinate direction); therefore, it is of interest to understand how uncertainty in specific features results in large position variability for these cases.

Previous studies have suggested that TC track is primarily a function of the deep-layer wind field [i.e., steering flow, e.g., George and Gray (1976)] and the advection of planetary vorticity by the TC circulation (e.g., Holland 1983). In general, the deep-layer steering wind is often closely related to the $500-700-\mathrm{hPa}$ winds (e.g., Chan and Gray 1982); however, individual cases can exhibit large variability in the steering flow depth (e.g., George and Gray 1976; Dong and Neumann 1986; Velden and Leslie 1991; Aberson and DeMaria 1994). Given that the wind speed and direction is often determined by the interaction of large-scale features, it is possible that the motion or structure of nearby synopticscale features could be associated with TC position errors (e.g., Carr and Elsberry 2000; Wu et al. 2004).

One method of evaluating the origin of TC position errors is through sensitivity analysis, which provides information about how small changes to the initial 
conditions can impact a forecast metric, such as TC position, at a particular time. Although position forecast sensitivity can exhibit large case-to-case variability and within methods (e.g., Majumdar et al. 2006; Wu et al. 2009; Hoover et al. 2013), previous studies have suggested that TC position forecasts can be sensitive to specific flow features, such as weaknesses in the subtropical ridge, the motion and evolution of midlatitude troughs, the position and speed of the subtropical jet, as well as uncertainty in the 0-h TC steering flow (e.g., Majumdar et al. 2006; Peng and Reynolds 2006; Wu et al. 2007; Chen et al. 2009; Wu et al. 2009; Komaromi et al. 2011; Gombos et al. 2012; Ido and $\mathrm{Wu}$ 2013; Nystrom et al. 2018). Furthermore, latent heat release associated with a TC and nearby convection can have an important impact on TC motion by modifying the nearby environment (e.g., $\mathrm{Wu}$ and Emanuel 1995a,b; Henderson et al. 1999; Anwender et al. 2008; Harr et al. 2008). In many of these cases, the TC is in close proximity to an upper-tropospheric potential vorticity (PV) anomaly. Furthermore, the divergent outflow from the convection can distort the PV field via advection (e.g., Archambault et al. 2013), leading to changes in the wind in the upper troposphere and hence the steering flow (e.g., Bassill 2014; Torn et al. 2015). As a consequence, it is not surprising that some TC position forecasts are sensitive to the distribution of latent heat release and divergent outflow.

One of the most difficult TC position forecasts appears to be associated with instances when the TC is located along the axis of contraction within a larger-scale deformation wind field. These situations can occur when a TC begins the process of extratropical transition (ET) and are often characterized by large position forecast variability due to the TC moving onto either side of the stagnation point (e.g., Grams et al. 2013; Riemer and Jones 2014). In these studies, the position sensitivity is determined by moving the TC to a new location in the model initial conditions, or by relaxing the initial conditions in specific regions around the TC (e.g., Nystrom et al. 2018). In the case of Hurricane Sandy, which was also located in a deformation flow, differences in convection (e.g., Bassill 2014; Torn et al. 2015) or small differences in the steering flow (e.g., Munsell and Zhang 2014) early in the forecast led to Sandy moving onto opposite sides of the axis of contraction, leading to dramatically different track forecasts. Nevertheless, most of these previous studies are individual case studies and employ different techniques to assess the source of the position variability, which makes it difficult to draw more general conclusions on position sensitivity in these instances. As a consequence, it is worthwhile to determine whether TC position forecasts within large-scale deformation steering flow are more sensitive to uncertainty in the evolution of remote features, such as midlatitude troughs or ridges, or if these forecasts are more sensitive to the steering flow associated with nearby features over a larger set of cases. Furthermore, it is important to quantify the role of initial position uncertainty, which is nonzero for most TCs (e.g., Torn and Snyder 2012; Landsea and Franklin 2013).

The goal of this study is to evaluate the role of remote and nearby steering flow uncertainty and initial position uncertainty on large position variability within European Centre for Medium-Range Weather Forecasts (ECMWF) ensemble forecasts of three cases [Debby (2012), Joaquin (2015), and Lionrock (2016)]. All three of these cases were characterized by highly anisotropic position variability within the first $72 \mathrm{~h}$ of the forecast and the $0-h$ TC position near the axis of contraction of a large-scale deformation wind field. The above hypotheses are evaluated by applying the ensemble-based sensitivity technique to the ECMWF ensemble forecasts to determine the relative contribution of nearstorm steering flow uncertainty relative to uncertainty in the wind farther from the TC.

The remainder of the paper proceeds as follows. Section 2 describes the dataset and methods used in this study. Section 3 provides a brief overview of the three cases, while section 4 describes the sensitivity of the position forecasts to the steering flow. A summary and conclusions are given in section 5 .

\section{Methods}

ECMWF TC track forecasts are evaluated for three cases characterized by significant anisotropic position variability and that are within a deformation steering flow pattern. ECMWF ensemble forecasts are employed here due to the size of the ensemble (51 members) and the good correspondence between ensemble-mean TC position errors and the ensemble standard deviation (e.g., Hamill et al. 2013). Gridded ECMWF forecast data at $0.5^{\circ}$ resolution are obtained from the THORPEX Interactive Grand Global Ensemble (TIGGE; Bougeault et al. 2010) archive located at ECMWF (http://apps. ecmwf.int/datasets/data/tigge), while TC tracking data are taken from the TIGGE TC archive (ftp://tigge:tigge@ tigge-ldm.ecmwf.int/cxml). Table 1 provides the initialization times of the cases used in this study as well as the operational ECMWF model version that was employed during this period.

TC position sensitivities at individual lead times are evaluated using the ensemble-based sensitivity method (Ancell and Hakim 2007; Torn and Hakim 2008) applied to the ECMWF ensemble data. The sensitivity pattern identified by this method yields the effect of a perturbation to a state variable onto the ensemble's forecast 
TABLE 1. Tropical cyclone name, initialization time, ECMWF version, optimal steering flow, and major axis unit vector associated with each case.

\begin{tabular}{lcccrr}
\hline \hline Tropical cyclone & Initialization time and date & ECMWF version & Steering layer (hPa) & Radius (km) & Major axis unit vector \\
\hline Debby (04L) & 0000 UTC 24 Jun 2012 & CY36R1 & $250-850$ & 333 & $0.968 \mathbf{i}, 0.249 \mathbf{j}$ \\
Joaquin (11L) & 0000 UTC 30 Sep 2015 & CY41R1 & $250-850$ & 333 & $-0.229 \mathbf{i}, 0.973 \mathbf{j}$ \\
Lionrock (12W) & 0000 UTC 27 Aug 2016 & CY41R2 & $200-850$ & 333 & $-0.731 \mathbf{i}, 0.682 \mathbf{j}$ \\
\hline
\end{tabular}

metric subspace, assuming that the ensemble has sufficient variability in both the state and metric (e.g., Gombos et al. 2012; Ido and Wu 2013). Specifically, the sensitivity of a forecast metric $(J)$ at time $t$ to a model state variable at location $i$ at an earlier lead time $\left(x_{i, t-\delta t}\right)$ is determined via

$$
\frac{\partial J}{\partial x_{i, t-\delta t}}=\frac{\operatorname{cov}\left(\mathbf{J}, \mathbf{x}_{i, t-\delta t}\right)}{\operatorname{var}\left(\mathbf{x}_{i, t-\delta t}\right)}
$$

where cov denotes covariance and var denotes variance. Similar to previous work, $\mathbf{x}_{i, t-\delta t}$ is normalized by its ensemble standard deviation prior to computing the sensitivity. This approach yields sensitivity values with units of the change in the forecast metric per standard deviation of the forecast field, which allows for a quantitative comparison between different forecast fields and lead times. The statistical significance of the sensitivity values is evaluated using the method outlined in Torn and Hakim (2008), which involves computing the $95 \%$ confidence bounds on the regression coefficient and testing the null hypothesis of no relationship between the metric and analysis state variable. If a regression coefficient is greater than the confidence bounds, the sensitivity value is said to be statistically significant.

Throughout the manuscript, $J$ will mainly be the distance along the horizontal axis that represents the greatest variability in TC position at a specific forecast lead time (hereafter referred to as the major axis). Essentially, this forecast metric represents the distance from the ensemble mean along the major axis of the position ellipse computed using the Hamill et al. (2011) methodology. Here, the major axis direction at a given lead time is determined by computing the eigenvectors of the zonal and meridional displacement from the ensemble-mean TC position based on the ensemble member positions. The benefit of this approach is that the position variability is not limited to the Cartesian coordinate framework, which is especially useful for cases where the axis of greatest variability has components in both the zonal and meridional directions. The orientation of the major axis is independently determined at each lead time.

TC steering flow is evaluated within each member of the ECMWF ensemble using the method outlined in Galarneau and Davis (2013), which is summarized below. This technique separates the TC vortex from the environmental steering flow by first computing the vorticity and divergence on individual pressure levels, then applying the Poisson equation to determine the streamfunction and velocity potential associated with

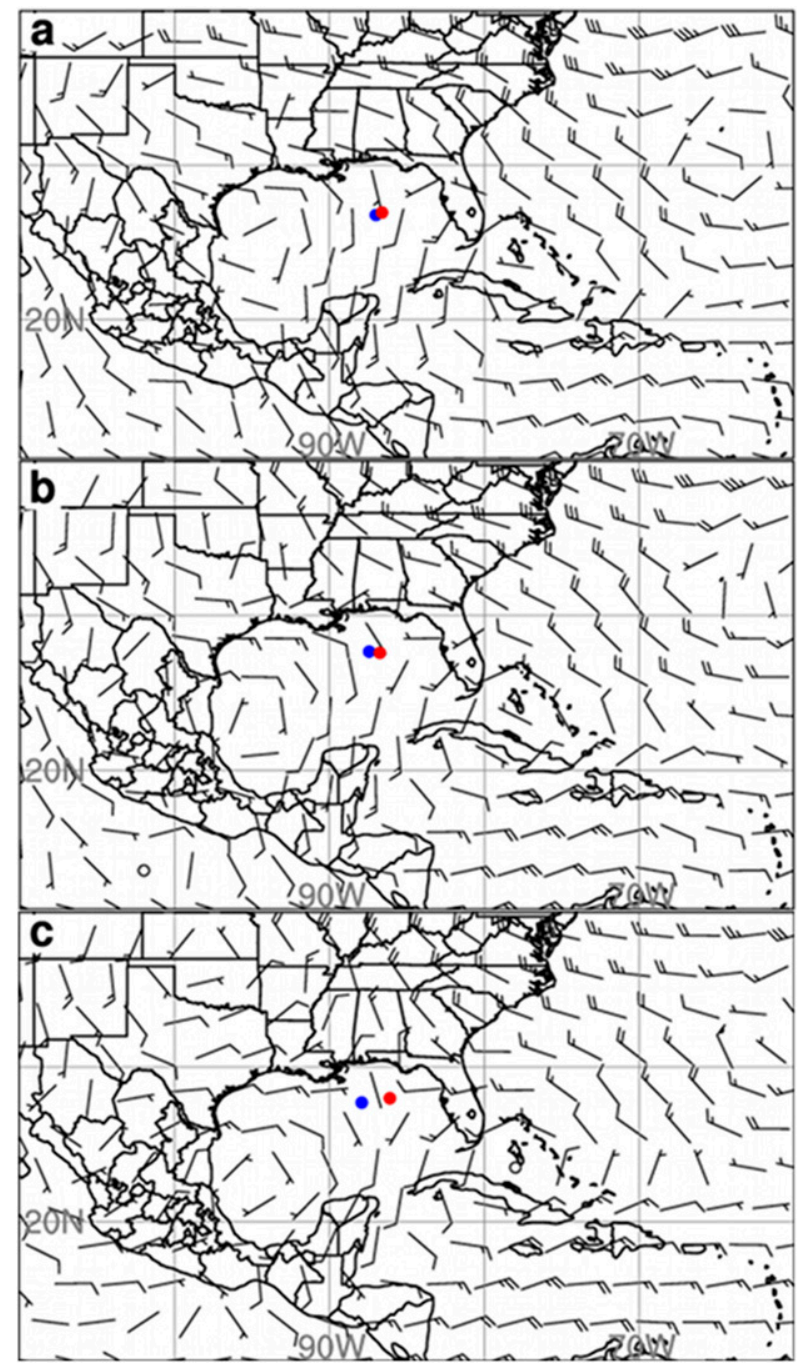

FIG. 1. ECMWF (a) 0-, (b) 12-, and (b) 24-h ensemble-mean steering winds for the forecast initialized at 0000 UTC 24 Jun 2012 (barbs). The red dot denotes the mean position of the 10 ensemble members with the most eastern 48 -h position, while the blue dot denotes the mean position of the 10 ensemble members with the most western 48 -h position. 


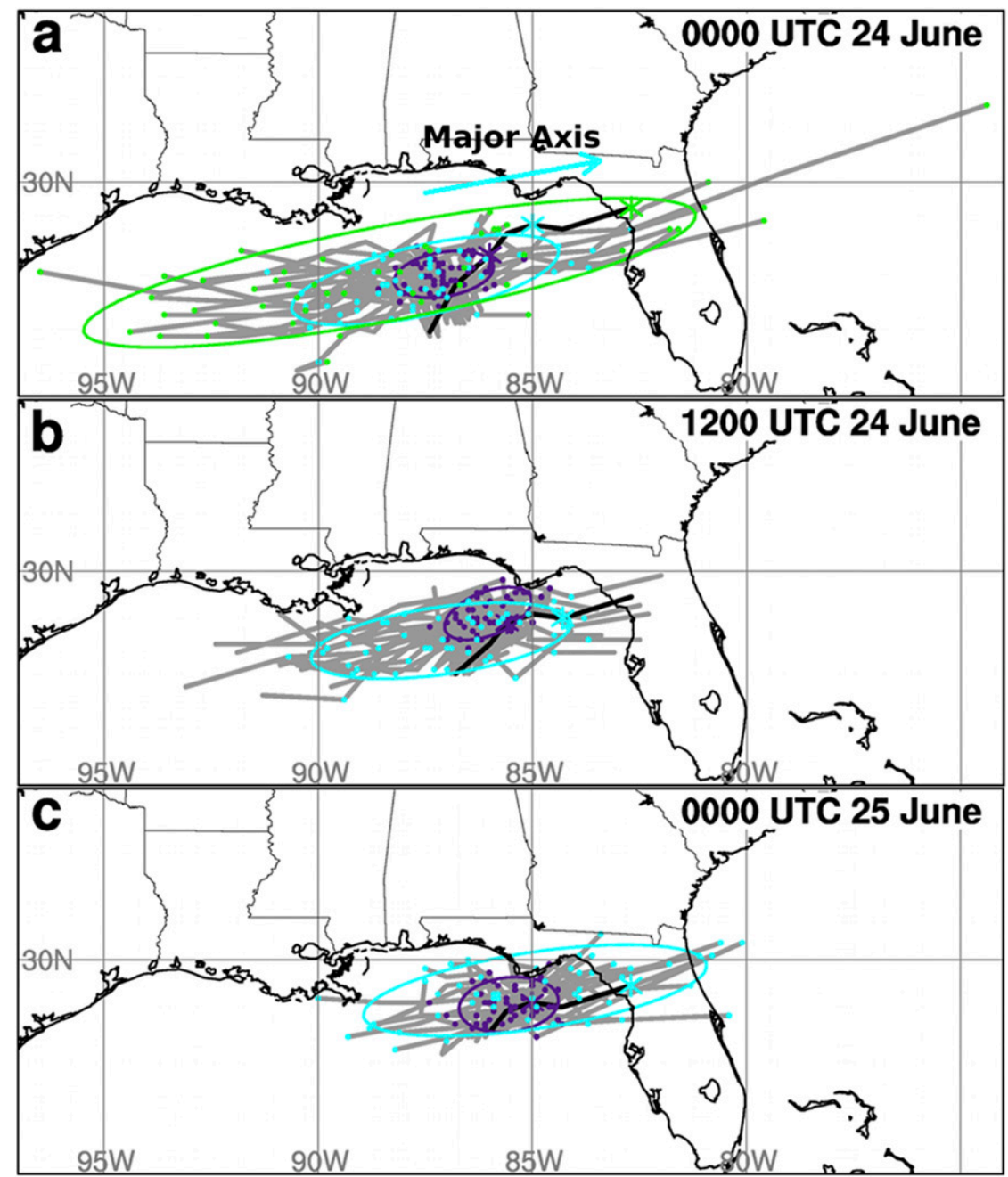

FIG. 2. ECMWF ensemble forecasts of Tropical Storm Debby initialized at (a) 0000 UTC 24 Jun, (b) 1200 UTC 24 Jun, and (c) 0000 UTC 25 Jun 2012 (gray lines). The dots indicate the location of each ensemble member at 24-h intervals, while the colored circles show a bivariate normal fit to the positions each $24 \mathrm{~h}$, as in Hamill et al. (2011). Purple denotes 24-h locations, cyan denotes 48 -h locations, and green denotes 72 -h locations. The thick black line denotes the NHC best track positions, while the stars indicate the corresponding best track position each $24 \mathrm{~h}$. The direction of the 48-h major axis is denoted by the cyan vector.

the vorticity and divergence within a given the radius of the TC center. The resulting nondivergent and irrotational winds are said to be associated with the TC vortex. From there, the vector environmental wind can be calculated by taking the difference between the vector wind and the nondivergent and irrotational vector wind associated with the TC vortex at a given horizontal location. The TC steering wind is then found by taking the mean of the environmental wind within a particular radius of the TC center and over a set of vertical layers starting at $850 \mathrm{hPa}$. The optimal steering flow is defined as the radius and vertical layer that is most similar to the TC motion within $\pm 12 \mathrm{~h}$ of a particular time. For all of the cases used here, the optimal steering flow (given in Table 1) is assumed to be the same for all members and lead times. This is done both for simplicity and because the optimal steering flow exhibited little variability over the important periods of time and in between individual ensemble members (not shown).

\section{Overview of cases}

\section{a. Tropical Storm Debby (2012)}

Tropical Storm Debby formed from the merger of a weak low pressure system that moved from the Gulf of Tehuantepec $\left(\approx 15^{\circ} \mathrm{N}, 95^{\circ} \mathrm{W}\right)$ into the western Caribbean 
Sea and the northern end of a tropical wave at 1200 UTC 23 June 2012. Subsequently, Debby moved northward through the central Gulf of Mexico along the axis of contraction of a deformation steering flow formed by a cyclonic circulation over the western Gulf of Mexico and eastern United States and an anticyclonic circulation over the south-central United States and Cuba (Fig. 1a). On 26 June, Debby turned to the east and made landfall along the coast of Florida at 2100 UTC. Over its 4-day lifetime, Debby was unable to intensify beyond a $55-\mathrm{kt}$ tropical storm (where $1 \mathrm{kt}=\sim 0.5144 \mathrm{~m} \mathrm{~s}^{-1}$ ) as a result of strong westerly vertical wind shear and midlatitude dryair intrusions. Instead, the biggest impacts from Debby were associated with rainfall; large regions of northcentral Florida recorded $>10 \mathrm{in} .(25.4 \mathrm{~cm})$ of rainfall over a 2-day period (Kimberlain 2013).

For initialization times close to genesis, Debby's position forecasts exhibited large variability, which in turn led to significant challenges for National Hurricane Center forecasters. Figure 2a shows the ECMWF ensemble forecasts initialized at 0000 UTC 24 June. At $0 \mathrm{~h}$, Debby is located in the central Gulf of Mexico near $27^{\circ} \mathrm{N}, 87.5^{\circ} \mathrm{W}$. While most of the ensemble members captured the slow northward drift during the first $24 \mathrm{~h}$, large differences between members exist thereafter, with some members exhibiting a south-of-west motion beyond that time, which results in Debby moving toward the western Gulf of Mexico. Other members exhibit continued slow northward motion into the northern Gulf of Mexico, while a third group follows the best track motion first toward the northeast and then east, making landfall along the Florida coast. This track forecast is characterized by a significant track bifurcation and resulted in a large 48-h NHC official forecast position error $(512 \mathrm{~km}$; $435 \%$ greater than the average 48-h NHC official position error over the previous $5 \mathrm{yr}$ ), since the official NHC forecast predicted Debby would take the more westward track.

\section{b. Hurricane Joaquin (2015)}

Hurricane Joaquin originated from a weak uppertropospheric low that developed over the eastern Atlantic Ocean during the middle of September. Over time, this feature gradually became a stronger cyclone, with deep convection developing on 27 September, leading to the designation of a tropical depression at 0000 UTC 28 September. Over the next 3 days, Joaquin moved toward the southwest in between an anticyclone located to its north along $70^{\circ} \mathrm{W}$, a cyclone to its east, and a deep anticyclone centered over Cuba (Fig. 3). During this time period, Joaquin underwent a period of rapid intensification, becoming a major hurricane at 0600 UTC 30 September. Between 1 and 2 October, an upper trough moved south and eastward from the United States leading

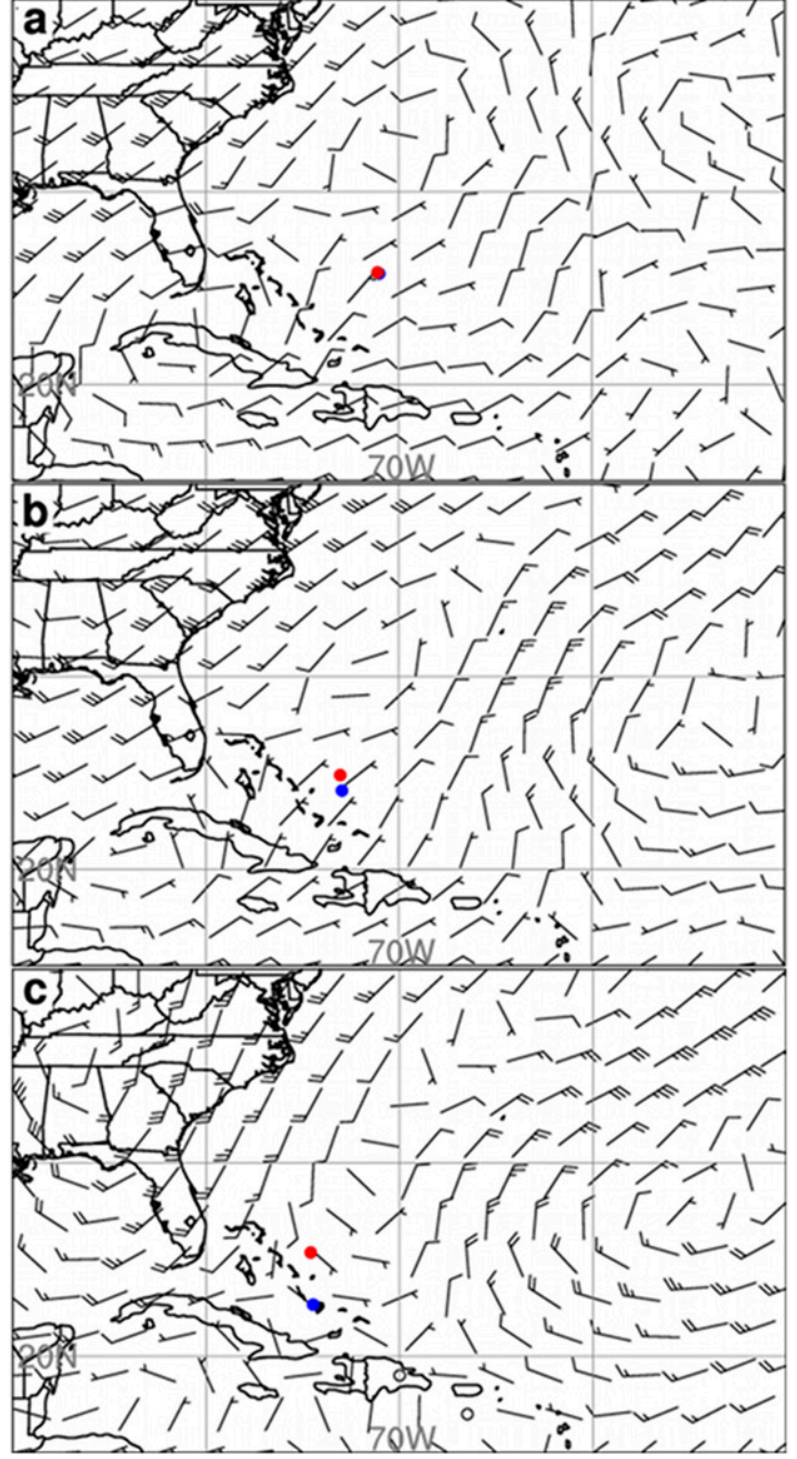

FIG. 3. ECMWF (a) 0-, (b) 24-, and (b) 48-h ensemble-mean steering winds for the forecast initialized at 0000 UTC 30 Sep 2015 (barbs). The red dot denotes the mean position of the 10 ensemble members with the most northern 72 -h position, while the blue dot denotes the mean position of the 10 ensemble members with the most southern 72-h position.

to the weakening of the ridge to the north, causing Joaquin to make a sharp clockwise turn in the Bahamas. As a consequence, Joaquin took on a more northeasterly motion for much of its remaining lifetime (Berg 2016).

ECMWF ensemble forecasts of Joaquin initialized at 0000 UTC 30 September exhibited considerable variability in the motion of the TC, which in turn provided a variety of landfall positions (Fig. 4a). During the first $24 \mathrm{~h}$, there was a significant amount of variability in the motion, with some members exhibiting a westerly direction of motion, while others were closer to the best track with 

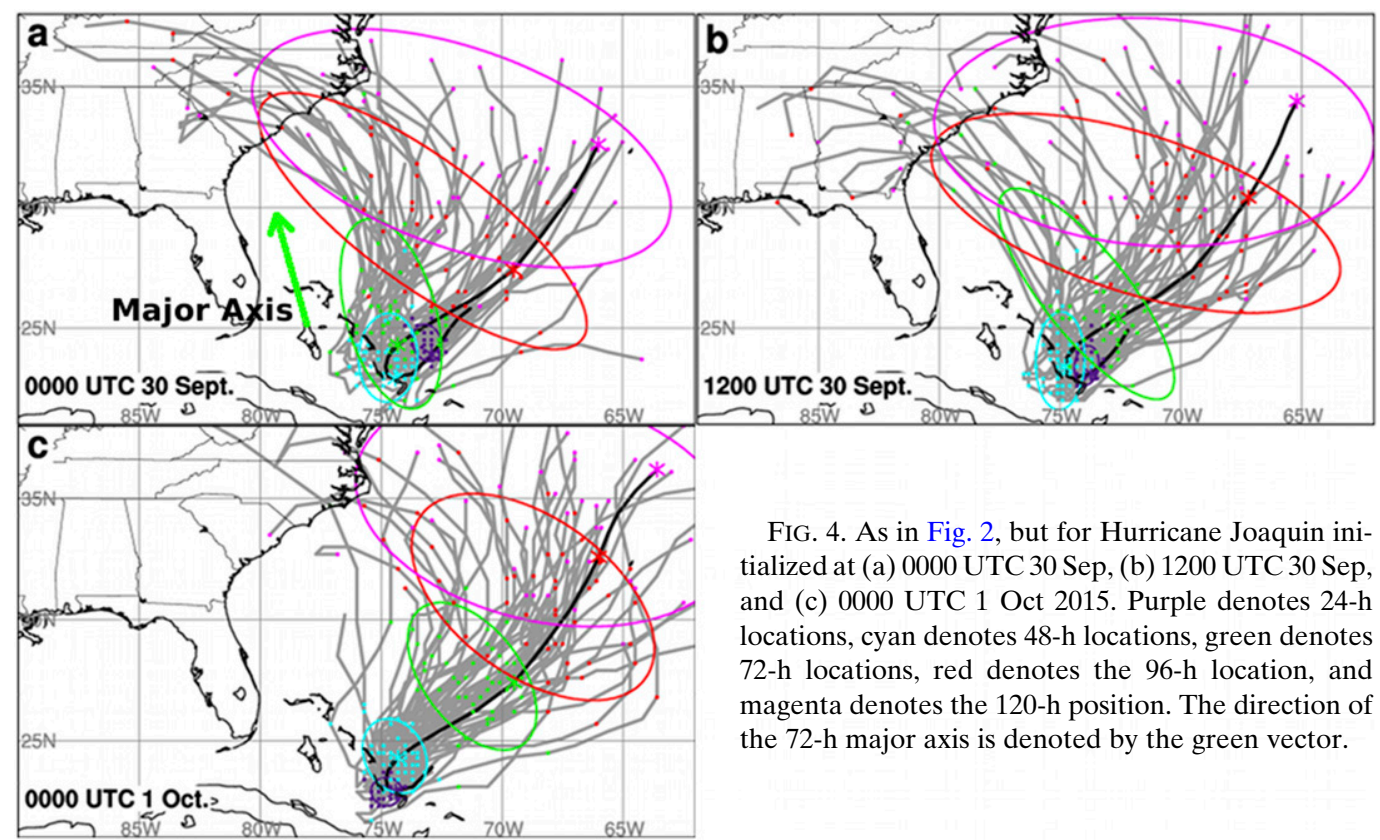

FIG. 4. As in Fig. 2, but for Hurricane Joaquin initialized at (a) 0000 UTC $30 \mathrm{Sep}$, (b) 1200 UTC $30 \mathrm{Sep}$, and (c) 0000 UTC 1 Oct 2015. Purple denotes 24-h locations, cyan denotes 48 -h locations, green denotes 72-h locations, red denotes the 96-h location, and magenta denotes the $120-\mathrm{h}$ position. The direction of the 72-h major axis is denoted by the green vector.

southwesterly movement toward the Bahamas. The latter set of members continued to move southwest between 24 and $48 \mathrm{~h}$, then turning around and moving to the northeast thereafter, similar to the best track. By contrast, the set of members that had the more westerly motion during the first $24 \mathrm{~h}$ acquired a more northerly motion between 48 and $72 \mathrm{~h}$ and a more northwesterly motion thereafter due to the aforementioned midlatitude trough that dug into the southeastern United States (Figs. 3b,c). In turn, this group predicted that Joaquin would make landfall in either North or South Carolina. Similar to Debby, the official NHC forecast was closer to the western motion subgroup, which resulted in a $96-\mathrm{h}$ position error of $522 \mathrm{~km}$.

\section{c. Typhoon Lionrock (2016)}

Typhoon Lionrock transitioned from a subtropical to tropical cyclone near $28^{\circ} \mathrm{N}, 154^{\circ} \mathrm{E}$ at $1800 \mathrm{UTC} 17$ August 2016. ${ }^{1}$ Over the next 6 days, Lionrock moved southwestward in response to a building subtropical high to the northwest and anticyclonic wave breaking to its east and intensified into a 95-kt typhoon by 0000 UTC 26 August, when the TC reached its farthest southwestern point. Beyond that time, the TC began to move to the northeast in response to a deep cyclonic circulation over northeast China and an anticyclone to its east, which combined to create a large-scale deformation steering flow pattern (Fig. 5). During this time, the typhoon reached its

\footnotetext{
${ }^{1}$ Genesis, position, and intensity based on Joint Typhoon Warning Center (JTWC) best track information.
}

maximum intensity of $120 \mathrm{kt}$ at 0000 UTC 28 August. By 0000 UTC 30 August, the typhoon turned sharply to the northwest in response to a deepening trough to its southwest as it underwent ET, leading to a rare landfall along Japan's eastern coast, which is similar to the motion of Hurricane Sandy along the East Coast of the United States in 2012 (Blake et al. 2013). The typhoon was associated with extensive damage both in Japan and North Korea, resulting in 550 deaths and $\$ 325$ million (U.S. dollars) in damage (Podlaha et al. 2016).

Although Lionrock's position forecast near the time of ET exhibited large position variability for many initialization times, the focus of this study will be in the ensemble forecast initialized at 0000 UTC 27 August (Fig. 6a), which is one of the last initialization times that contains significant across-track variability at the time of ET. During the first $48 \mathrm{~h}$, the ensemble standard deviation in across-track position is less than $100 \mathrm{~km}$; however, by $72 \mathrm{~h}$, the ensemble positions become highly anisotropic, with some members showing Lionrock in the Sea of Japan, moving quickly north of due west around a midlatitude cyclone, while another set of members has Lionrock continuing to move to the northeast at a slower rate. The position variability for this case resembles the ensemble forecasts for Hurricane Sandy (e.g., Torn et al. 2015).

\section{Results}

\section{a. Tropical Storm Debby (2012)}

Given the large position variability in this forecast, it is of interest to understand what processes contributed 


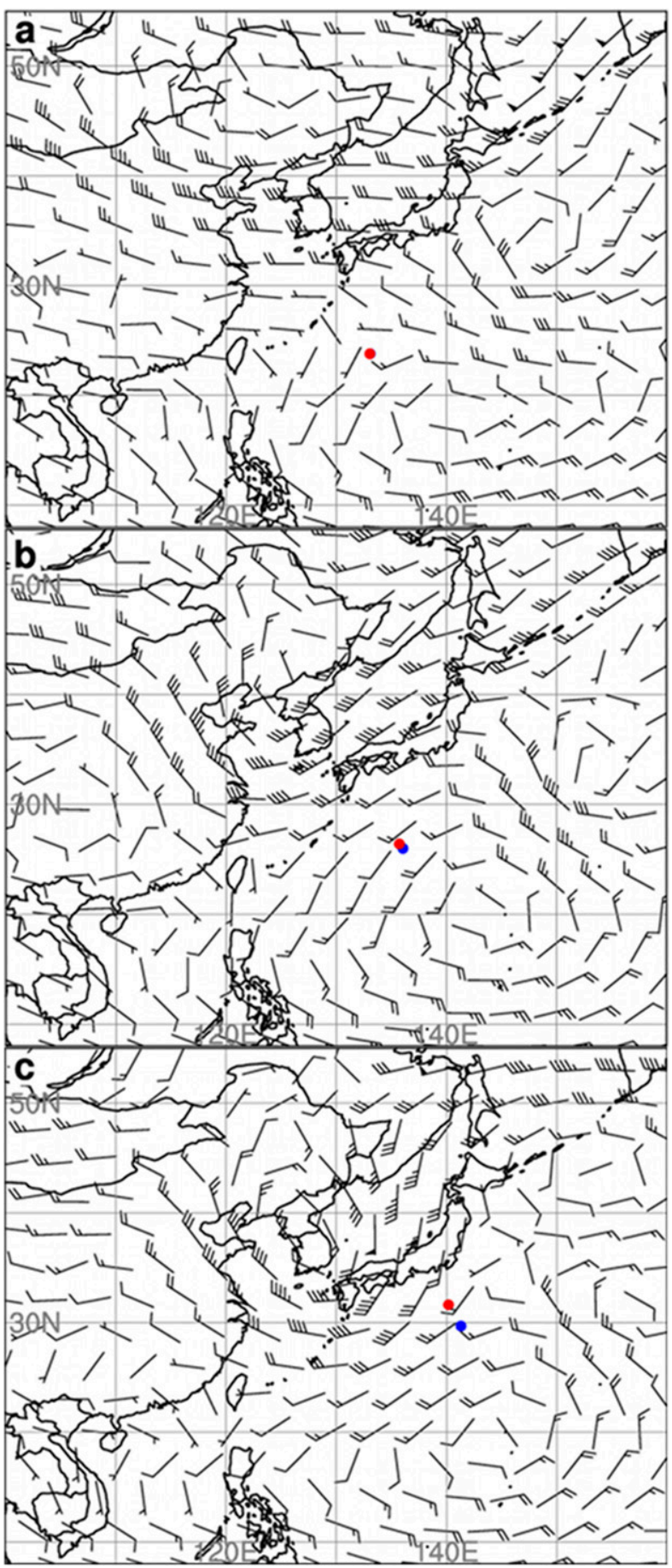

FIG. 5. ECMWF (a) 0-, (b) 24-, and (b) 48-h ensemble-mean steering winds for the forecast initialized at 0000 UTC 27 Aug 2016 (barbs). The red dot denotes the mean position of the 10 ensemble members with the most northwestern 72 -h position, while the blue dot denotes the mean position of the 10 ensemble members with the most southeastern $72-\mathrm{h}$ position. to the highly anisotropic position variability associated with this case. One hypothesis for the large 48-h position variability is that it is a consequence of position variability at earlier forecast lead times. TC position errors can be thought of as the integral of the steering flow errors over time (i.e., a TC subjected to an erroneous $1 \mathrm{~m} \mathrm{~s}^{-1}$ westerly wind would result in a 86-km easterly position error by 1 day); therefore, it is likely that the 48 -h position variability is correlated to position variability at earlier times. Figure 7a shows the correlation between Debby's 48-h position along the major axis to the distance along the major axis at earlier lead times. At $0 \mathrm{~h}$, the correlation is 0.37 (statistically significant at the $95 \%$ confidence level), which increases to 0.86 by $24 \mathrm{~h}$. This result suggests that the large variability in Debby's 48-h position forecast is strongly related to position differences that develop within the first $24 \mathrm{~h}$ of the forecast. In turn, this suggests that the initial position and steering flow uncertainty is important.

Prior to understanding the role of 0 -h steering flow differences, it is necessary to determine the appropriate steering flow for Debby over this period. Figure 8a shows the mean absolute difference between the steering flow computed using the variety of depths and radii using the Galarneau and Davis (2013) method and the best track motion averaged over each ensemble member and lead time during the first $24 \mathrm{~h}$. For these times, the minimum in mean absolute error (MAE) and the minimum standard deviation on motion differences occur when the steering flow is computed between 250 and $850 \mathrm{hPa}$ and the TC removal radius is $333 \mathrm{~km}$; therefore, the steering flow is assumed to be these parameters for the remainder of this section. Note that computing the steering flow differences for individual members yields similar values of the optimal steering flow depth and radius (not shown); therefore, the track differences are not due to differences in the steering flow for each member.

With the steering flow established, it is possible to evaluate how the uncertainty in the steering flow at various lead times correlates to the 48 -h position differences. Figure 7 a shows the correlation between the 48-h distance along the major axis and the component of the TC steering flow in the direction of the 48-h major axis as a function of lead time (unit vector given in Table 1). The correlation between the 0-h steering flow and 48-h position is 0.46 and increases with lead time, suggesting that early lead-time steering flow differences are strongly related to the subsequent position differences.

Steering flow is the vertically integrated wind; thus, it is possible that the uncertainty in the steering flow is mainly associated with uncertainty in the wind in a particular vertical layer of the atmosphere (i.e., upper or midtroposphere). Therefore, it is worth understanding 

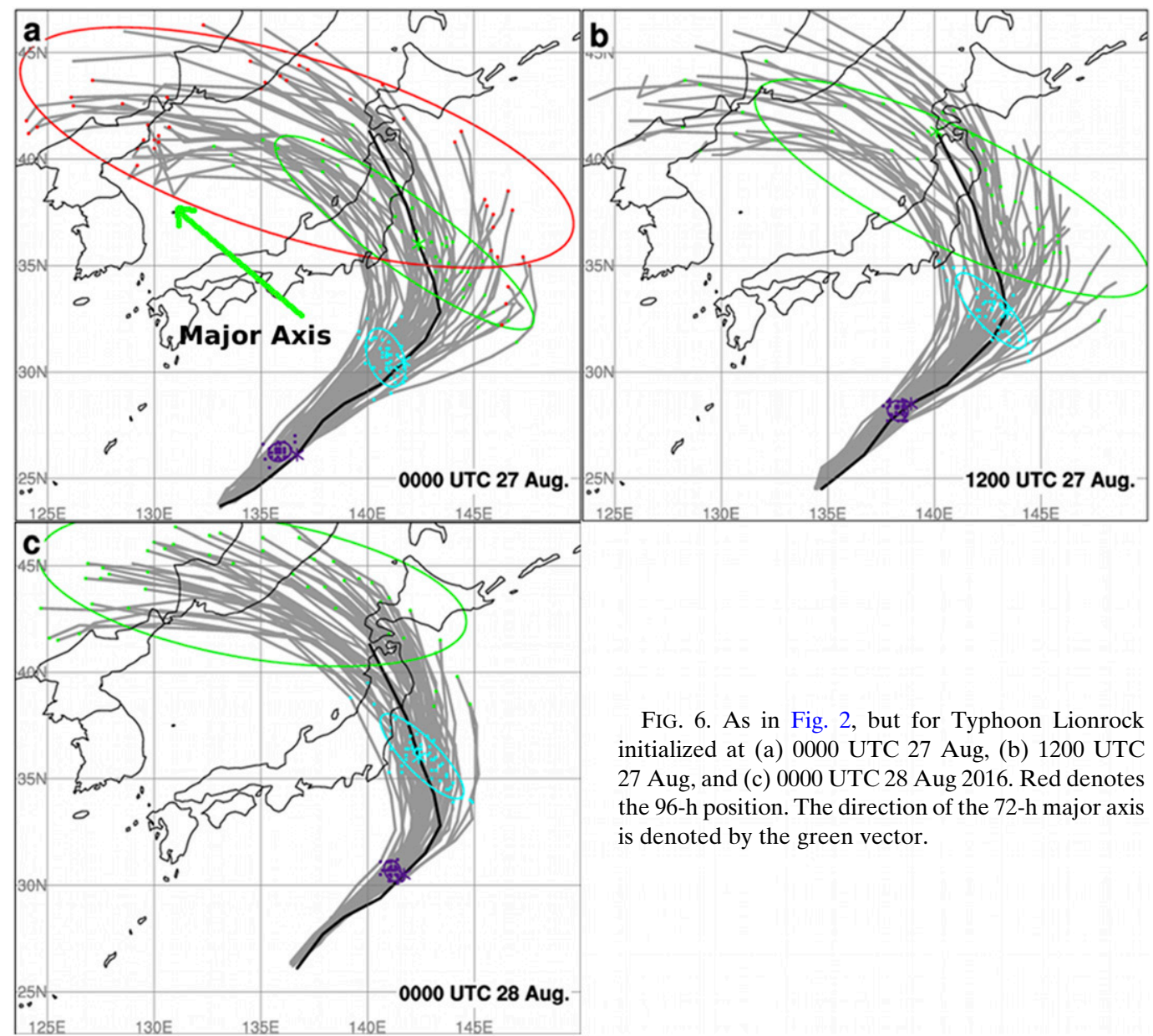

FIG. 6. As in Fig. 2, but for Typhoon Lionrock initialized at (a) 0000 UTC 27 Aug, (b) 1200 UTC 27 Aug, and (c) 0000 UTC 28 Aug 2016. Red denotes the $96-\mathrm{h}$ position. The direction of the 72 -h major axis is denoted by the green vector.

how changes to the steering flow at one vertical level contribute to the variability of the column-integrated steering flow. This is evaluated by computing the linear regression coefficient between the 0-h component of the TC steering flow in the direction of the major axis and the 0 -h component of the wind in the direction of the major axis at each vertical level (normalized by the ensemble standard deviation; Fig. 9a). Here, the TC steering wind at each level is computed in the same manner as the column-integrated steering flow (i.e., the TC vortex is removed). Vertical levels with a large regression coefficient denote where the column-integrated TC steering flow is most sensitive to changes in the wind at that level. At this time, the column-integrated steering flow is most sensitive to variability in the 500$700-\mathrm{hPa}$ wind $\left(0.22 \mathrm{~m} \mathrm{~s}^{-1}\right.$ per standard deviation), with comparatively less sensitivity to the upper-troposphere wind; other lead times exhibit a qualitatively similar sensitivity profile (not shown).

Given the strong correlation between the 48-h position variability and 0 -h steering flow, it is of interest to understand the sensitivity of the 48-h position forecast to the 0 -h steering flow as a function of space. Figure 10 shows that Debby is initially located within a region characterized by positive sensitivity that extends along the axis of contraction (located along roughly $88^{\circ} \mathrm{W}$ ) and the axis of dilatation (located along roughly $30^{\circ} \mathrm{N}$ ) of the deformation flow over the Gulf of Mexico. Here, positive sensitivity indicates that increasing the component of the steering flow in the direction of the 48-h major axis (i.e., a more westerly wind) by one standard deviation would result in Debby being $80 \mathrm{~km}$ to the east of the 48 -h ensemble-mean position, while the opposite is true for an easterly perturbation. In essence, this result suggests that perturbation easterly or westerly flow at the location of Debby would cause the TC to move onto either the western or eastern side of the steering flow axis of contraction, which in turn leads to the TC moving even farther away from the ensemble-mean position (demonstrated below).

The steering flow differences appear to be tied to uncertainty in the synoptic features surrounding Debby. Figure $10 \mathrm{~b}$ indicates that the 48 -h position forecast is 

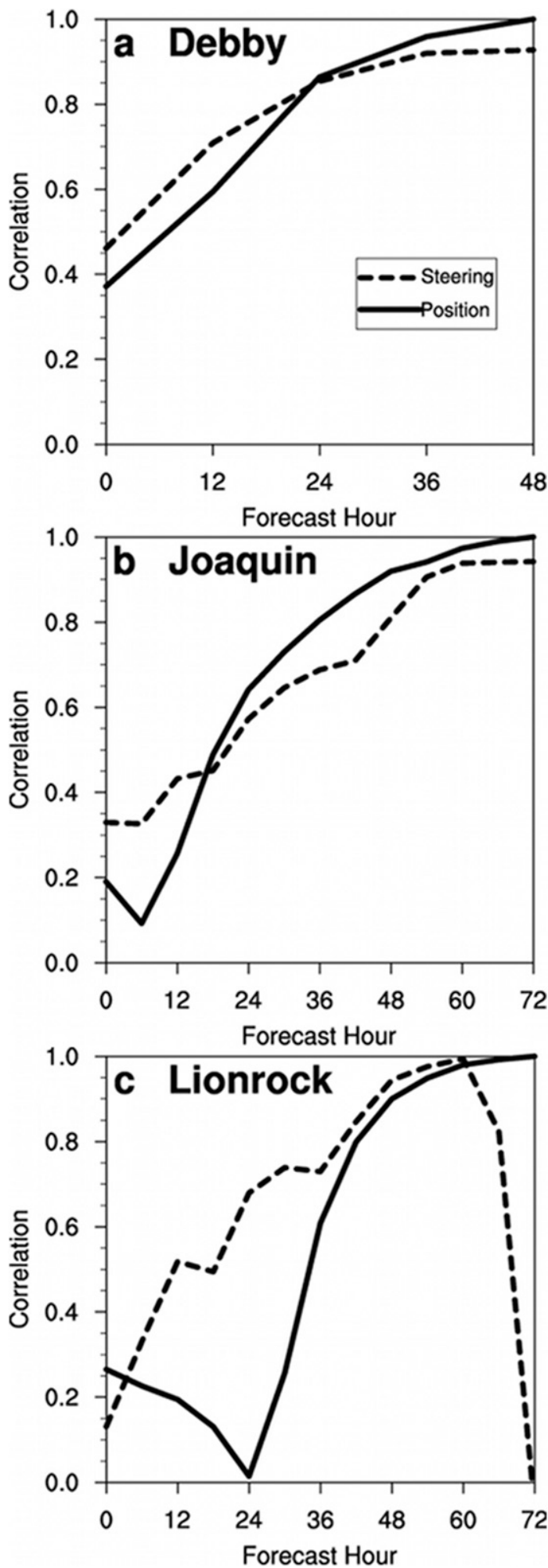

FIG. 7. (a) Correlation between Debby's 48-h distance along the major axis to the distance along the major axis at earlier forecast hours (solid line) initialized at 0000 UTC 24 Jun 2012. The dashed line indicates the correlation between Debby's 48-h distance along the major axis to the component of the steering flow in the direction sensitive to the 0 -h $500-\mathrm{hPa}$ geopotential height to the south of Debby over the western Caribbean and to the northeast of Debby over the southeastern United States. This dipole pattern of sensitivity indicates that increasing the heights to the south and/or decreasing the heights to the north, which in turn would yield a westerly geostrophic wind at Debby's 0-h position, results in a more eastern position later in the forecast. As a consequence, it appears that Debby's position forecast is most sensitive to the steering wind near the TC and not to the evolution of wind errors that originate farther away. Furthermore, the 48-h position forecast exhibits similar sensitivity to the steering flow and 500-hPa heights up to $36 \mathrm{~h}$ (not shown); thus, the sensitivity signal is robust.

The prior results suggest that Debby's position forecast is sensitive to the perturbation steering flow near the TC; however, the standard deviation in the component of the 0 -h steering flow in the direction of the 48-h major position axis is $0.27 \mathrm{~m} \mathrm{~s}^{-1}$. Assuming the steering wind standard deviation remains constant with time, it would yield a position difference of $23 \mathrm{~km} \mathrm{day}^{-1}$; therefore, the advection of the TC by the perturbation steering flow alone cannot explain the 191-km 48-h Debby position standard deviation. Instead, the position differences could be related to differences in the ensemble-mean steering flow, particularly since the TC is located within a deformation wind pattern, meaning that the perturbation wind could advect the TC into a region with a different ensemble-mean steering wind. Here, the contribution of the ensemble-mean and perturbation steering wind differences is quantified by partitioning the component of the steering wind in the direction of the 48-h major axis into an ensemble-mean and deviation from the ensemble mean (computed at Debby's position in each ensemble member) at each forecast lead time. The ensemble-mean and perturbation steering wind components are then averaged for the 10 members with the most eastern and western 48-h positions. The statistical significance of the differences between these two groups of members is evaluated by randomly resampling two sets of 10 members from the full 51-member ensemble 5000 times and determining the $95 \%$ confidence bounds, similar to what is done in Torn et al. (2015).

The steering flow differences between the western and eastern members of the ensemble show a general transition from differences that are dominated by the perturbation wind to differences dominated by the ensemble mean wind. Initially, the eastern members are

of the major axis at each lead time. (b) As in (a), but for Joaquin's 72-h major axis position initialized at 0000 UTC $30 \mathrm{Sep} 2015$. (c) As in (a), but for Lionrock's 72-h major axis position for the forecast initialized at 0000 UTC 27 Aug 2016. 

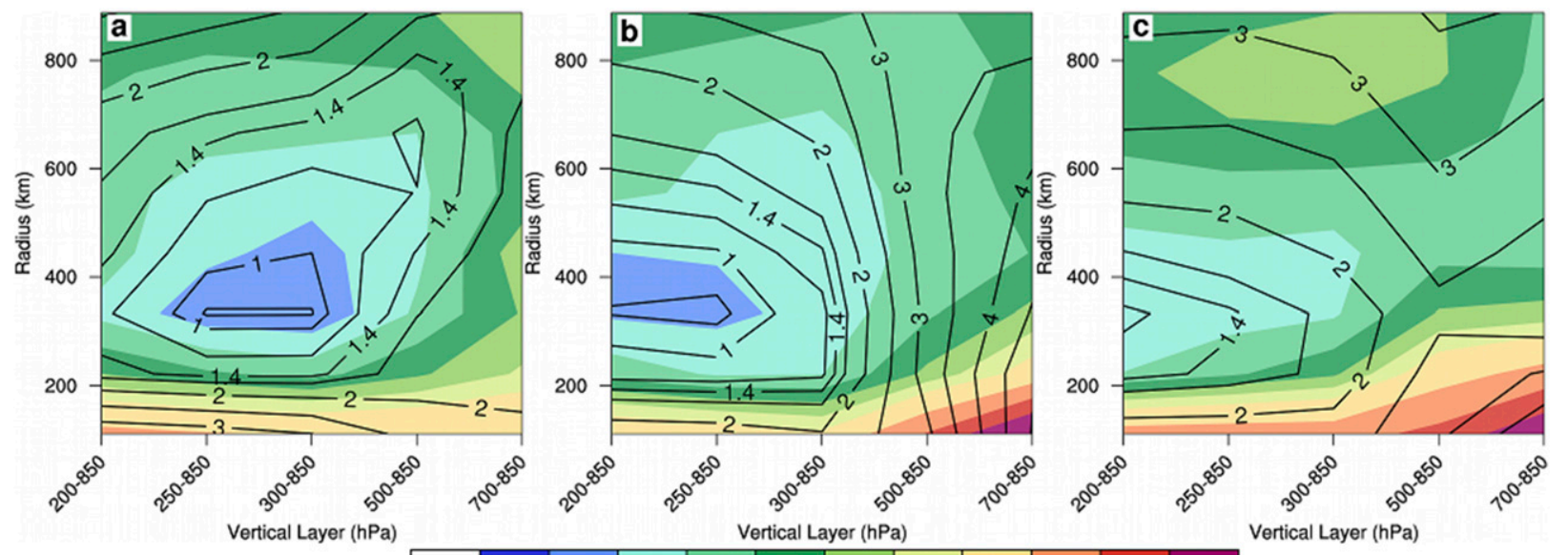

$\begin{array}{lllllllllll}0.3 & 0.4 & 0.5 & 0.6 & 0.7 & 0.8 & 0.9 & 1 & 1.2 & 1.4 & 1.6\end{array}$

FIG. 8. (a) Mean absolute vector wind difference between the motion of Debby and the environmental flow as a function of TC removal radii and vertical depths averaged between 0 - and 24-h lead time and over all ensemble members for the forecast initialized at 0000 UTC 24 Jun 2012 (contours, $\mathrm{m} \mathrm{s}^{-1}$ ). The shading denotes the standard deviation in the vector wind difference over all members and times. (b) As in (a), but for Joaquin's motion between 0 and $24 \mathrm{~h}$ initialized at 0000 UTC 30 Sep 2015. (c) As in (a), but for Lionrock's motion between 0 and $36 \mathrm{~h}$ initialized at 0000 UTC 27 Aug 2016.

characterized by a $0.2 \mathrm{~m} \mathrm{~s}^{-1}$ westerly perturbation steering wind, while the western members are characterized by a $0.2 \mathrm{~m} \mathrm{~s}^{-1}$ easterly perturbation steering wind (statistically significant difference at the $95 \%$ level), while the ensemble-mean steering wind is comparable between the members (Fig. 11a). By $12 \mathrm{~h}$, the difference in the perturbation steering flow between the eastern and western members increases to $0.6 \mathrm{~m} \mathrm{~s}^{-1}$; however, because the eastern members are now east of the axis of contraction (Figs. 1b,c), the ensemble-mean steering flow is westerly, while the western members are characterized by an easterly component (difference statistically significant). For the remainder of the forecast, the ensemble-mean steering flow becomes increasingly westerly for the eastern members and easterly for the western members, with the differences increasing to nearly $5.0 \mathrm{~m} \mathrm{~s}^{-1}$ by $48 \mathrm{~h}$. These results suggest that the track differences are the result of a two-step process. During the first $12 \mathrm{~h}$ of the forecast, the perturbation wind leads to Debby moving either slightly to the west or east. In turn, Debby moves onto either side of the axis of contraction of the deformation wind field, which causes the TC to experience a more westerly or easterly ensemble-mean steering flow. The differences in the mean steering flow are much larger than the perturbation steering wind differences, which subsequently lead to the large divergence in position forecasts beyond $48 \mathrm{~h}$.
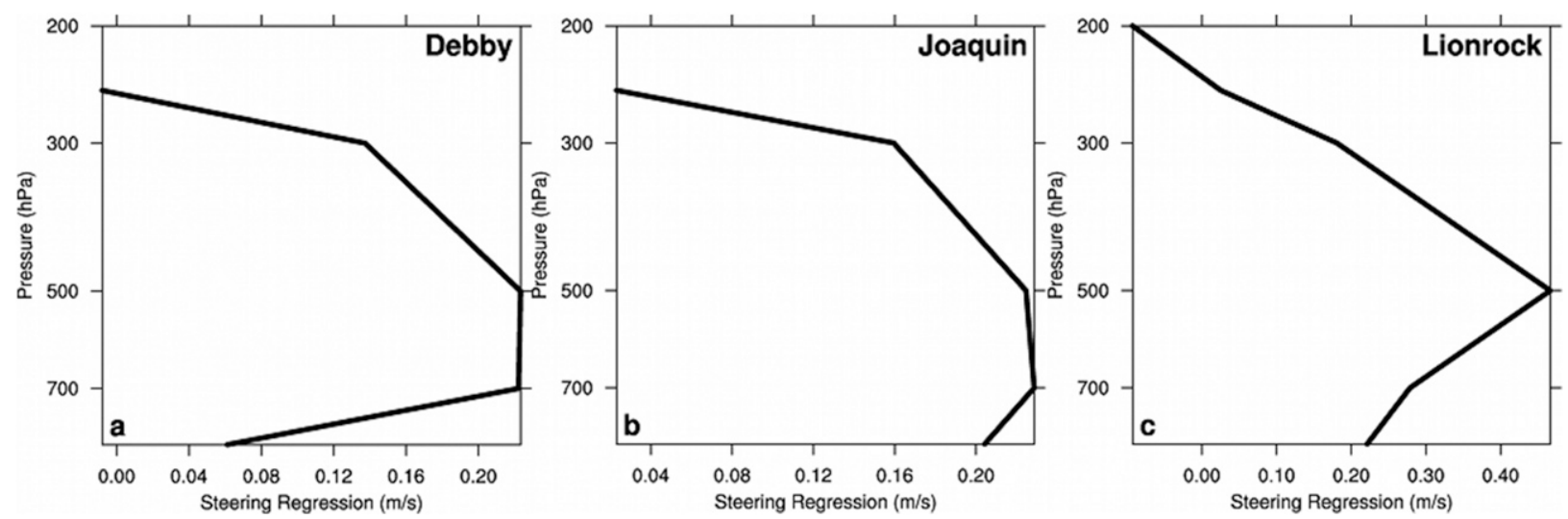

FIG. 9. (a) Change in the 0-h component of the steering wind along the 48-h major axis due to a one standard deviation change in the component of the steering wind along the major axis at each pressure level for the Debby forecast initialized at 0000 UTC 24 Jun 2012 ( $\mathrm{m} \mathrm{s}^{-1}$ per standard deviation). (b) As in (a), but for the 12-h Joaquin forecast initialized at 0000 UTC 30 Sep 2015. (c) As in (a), but for the 12-h Lionrock forecast initialized at 0000 UTC 27 Aug 2016. 


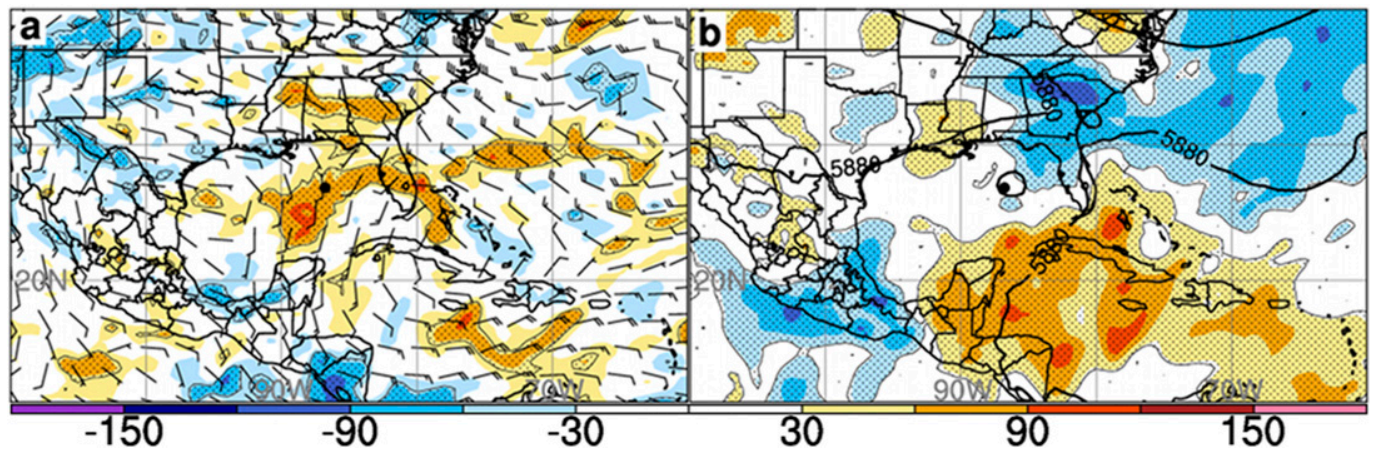

FIG. 10. Sensitivity of Debby's 48-h distance along the major axis to the 0 -h (a) component of the steering wind in the direction of the 48-h major axis and (b) the 500-hPa height (shading, $\mathrm{km}$ ). Stippled regions indicate where the sensitivity is statistically significant at the $95 \%$ confidence level. The barbs in (a) denote the ensemble-mean steering wind, while the contours in (b) denote the ensemble-mean 500-hPa heights (m). The large dot denotes Debby's 0-h position.

Forecasts initialized 12 and $24 \mathrm{~h}$ later support the notion that Debby's large position variability can be explained by the location relative to the axis of contraction. Ensemble forecasts initialized at 1200 UTC 24 June (12h later) exhibit a greater number of members that contain the eastern solution, with only eight members exhibiting a position to the west of $90^{\circ} \mathrm{W}$, which suggests that the ensemble narrows onto the eastern solution (Fig. 2b). Moreover, ensemble forecasts initialized at 0000 UTC 25 June have no members west of $90^{\circ} \mathrm{W}$, with additional members closer to the best track position (Fig. 2c). Over these three forecast cycles, there is a significant reduction in both the ensemble standard deviation and ensemble-mean error at a given verification time (0000 UTC 26 June; Fig. 12a). In particular, the ensemble standard deviation decreases by a factor of 3 , while the ensemble-mean error is $79 \%$ lower. This likely occurs because at later initialization times, it becomes clear that Debby will move to the right of the axis of contraction, which significantly reduces the range of possible position forecasts that can exist within the ensemble.

\section{b. Hurricane Joaquin (2015)}

A similar analysis is carried out on the Joaquin forecast to determine how the various synoptic features influenced the position forecast. Here, the focus is on the 72-h position forecast since subsequent position forecasts are highly dependent on the position at this time. For this particular initialization time, Joaquin's 72-h distance along the major axis (and hence the subsequent position forecasts) is strongly determined by its position variability within the first $24 \mathrm{~h}$ (Fig. $7 \mathrm{~b}$ ). Unlike the Debby case, the correlation between Joaquin's 72-h
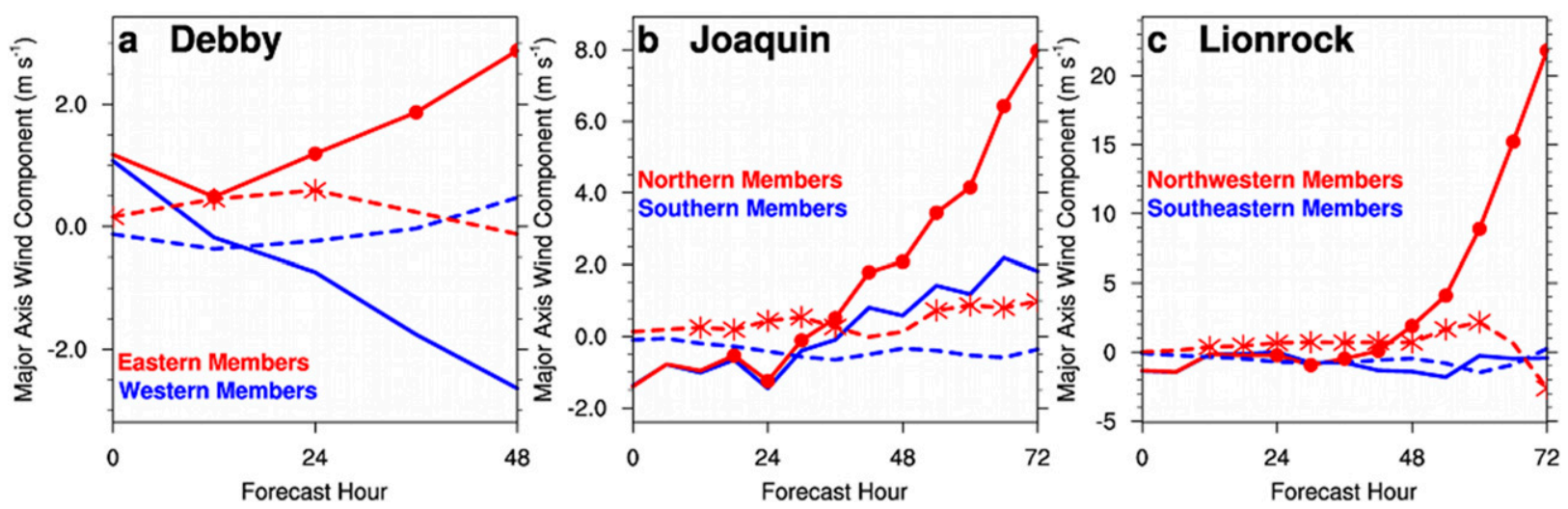

FIG. 11. (a) Ensemble-mean (solid) and ensemble perturbation (dashed) steering wind for TS Debby in the direction of the 48-h major axis for the 10 most western members (blue) and 10 most eastern members (red) at $48 \mathrm{~h}$ as a function of lead time for the forecast initialized at 0000 UTC 24 Jun 2012. Dots and stars denote times where the differences between the mean and perturbation wind are statistically significant at the $95 \%$ confidence level, respectively. (b) As in (a), but for the difference in the component of the steering flow along the 72-h major axis for Joaquin initialized at 0000 UTC 30 Sep 2015. (c) As in (a), but for the difference in the component of the steering flow along the 72-h major axis for Lionrock initialized at 0000 UTC 27 Aug 2016. 

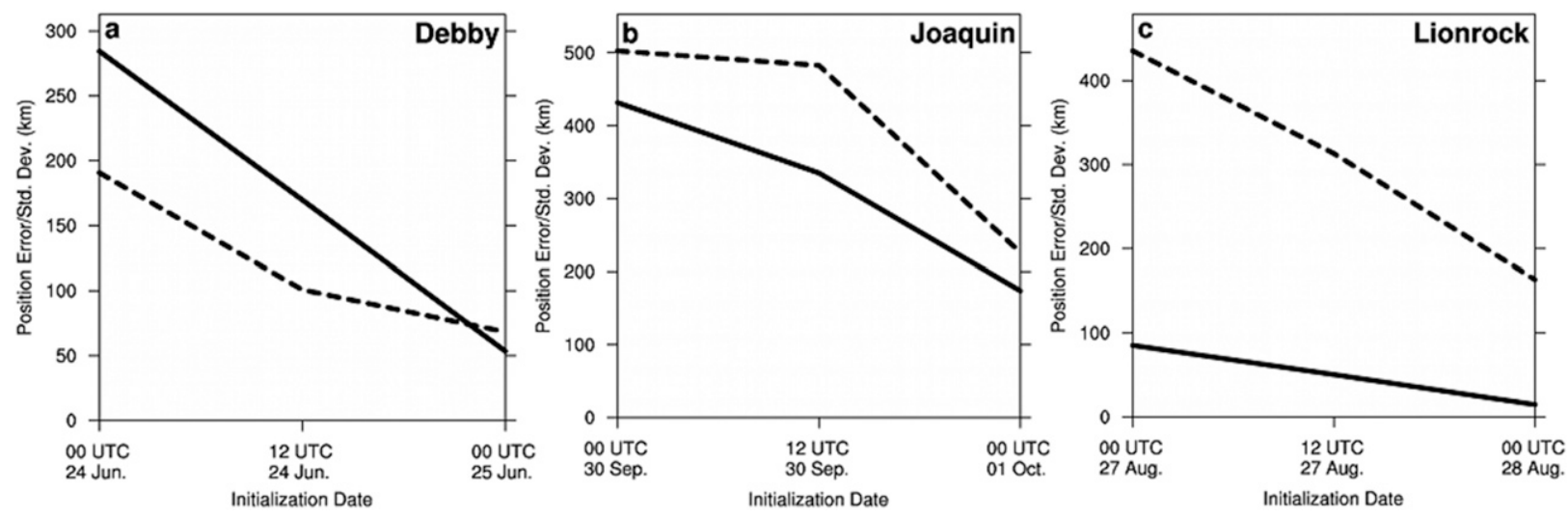

FIG. 12. (a) Ensemble-mean position error (solid) and ensemble standard deviation (dashed) in position for forecasts of Debby valid at 0000 UTC 26 Jun 2012 as a function of initialization time. (b) As in (a), but for forecasts of Joaquin valid at 0000 UTC 3 Oct 2015 . (c) As in (a), but for forecasts of Lionrock valid at 0000 UTC 30 Aug 2016.

position forecast and the $0-12$-h forecast is less than 0.24 (not statistically significant at the $95 \%$ level). Beyond $12 \mathrm{~h}$, the correlation increases to above 0.60 starting at $24 \mathrm{~h}$. As a consequence, it appears that the main position differences in Joaquin's forecast occur during the first $24 \mathrm{~h}$ of the forecast; therefore, it is important to focus on this time period and, in particular, on the role of variability in the steering flow.

Not surprisingly, the 72-h position forecast uncertainty in the direction of the major position variability (here roughly north-south; unit vector given in Table 1) exhibits a statistically significant correlation with the component of the steering flow of the major axis for all lead times. During the 0-24-h period, the optimal steering flow is defined using the $250-850-\mathrm{hPa}$ wind with a $333-\mathrm{km}$ TC removal radius (Fig. 8b), which is consistent for all ensemble members (not shown). In particular, the correlation with the steering flow increases from 0.33 to 0.57 during the first $24 \mathrm{~h}$, suggesting that the members that move farther to the south are characterized by greater northerly winds from 0 to $24 \mathrm{~h}$. In addition, variability in the component of the 12-h steering flow in the direction of the major axis is most sensitive to variability in the 500 700 -hPa wind $\left(0.23 \mathrm{~m} \mathrm{~s}^{-1}\right.$ change in the column-integrated steering wind per standard deviation in the wind at that level; Fig. 9b). In turn, this result suggests that uncertainty in the midtropospheric wind might explain the differences between the different ensemble members (other times indicate similar steering flow sensitivity; not shown).

The 72-h position forecast along the major axis appears to be most sensitive to the steering wind near the TC during the first $12 \mathrm{~h}$. Figure 13a indicates that Joaquin's position forecast is most sensitive to the 0 -h steering wind approximately $1.5^{\circ}$ to the west of the initial position, which is on the northeastern side of the deep-tropospheric anticyclone centered over Cuba and on the southwestern side of the anticyclone to the north of Joaquin. Within this region, making the wind more southerly by one standard deviation yields a 72-h position forecast that is roughly $140 \mathrm{~km}$ farther north than the ensemble mean. Over time, the region of maximum sensitivity drifts toward the southwest where Joaquin is moving, such that Joaquin moves into the middle of the sensitive region by $12 \mathrm{~h}$ (Fig. 13c). It is worth pointing out the region of negative sensitivity that is present at both times over the southeastern Gulf of Mexico and the western Caribbean Sea, which is on the western side of the Cuba anticyclone. The combination of positive sensitivity on the eastern side of the anticyclone and negative sensitivity on the western side implies that Joaquin's track forecast could be sensitive to the large-scale synoptic wind pattern in this area. Indeed, Figs. 13b and 13d indicate that Joaquin's position forecast is sensitive to the $500-\mathrm{hPa}$ heights within the deeptropospheric anticyclone and to the heights to the northeast of the TC, such that decreasing the heights with the Cuba anticyclone and increasing the heights associated with the midlatitude trough to the northeast, which would imply southerly geostrophic winds near Joaquin, is associated with a more northern TC later on in the forecast. Similar to Debby, these results suggest that Joaquin's forecast is most sensitive to the steering flow near the TC and not to steering flow uncertainty initially far away, such as the trough moving in from the southeast United States. Furthermore, this sensitivity is consistent with the results of Nystrom et al. (2018), who found that WRF forecasts of Joaquin appeared to be most sensitive to changing the initial conditions $600-900 \mathrm{~km}$ from the TC.

The ensemble position differences appear to be related to the combination of both perturbation and ensemble-mean steering flow differences among the members. Figure 11b shows the composite ensemblemean and perturbation steering wind components in the 


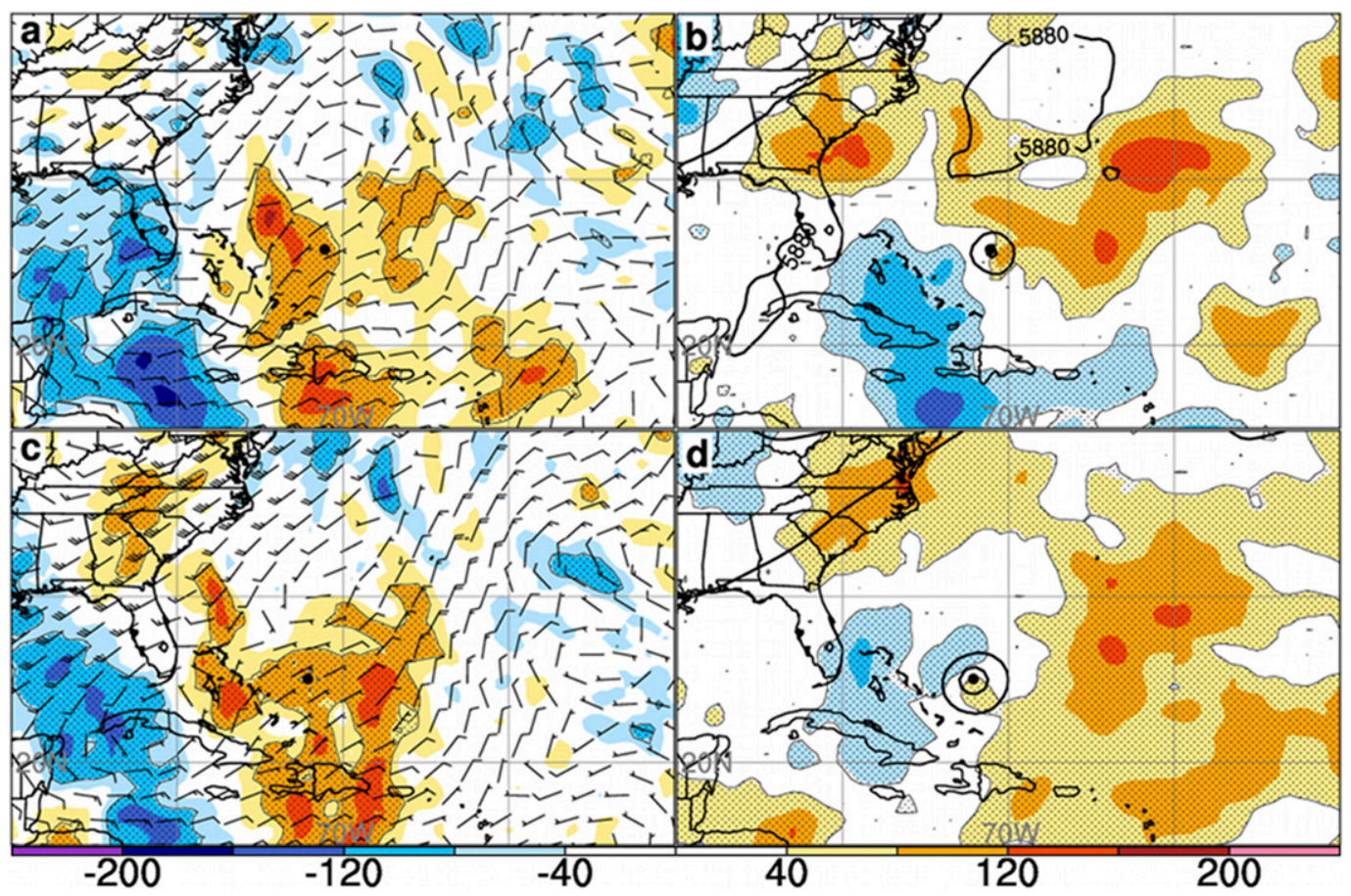

FIG. 13. Sensitivity of Joaquin's 72-h distance along the major axis to the 0 -h (a) component of the steering wind in the direction of the 72-h major axis and (b) the 500-hPa height (shading, $\mathrm{km}$ ). Stippled regions indicate where the sensitivity is statistically significant at the $95 \%$ confidence level. The barbs in (a) denote the ensemble-mean steering wind, while the contours in (b) denote the ensemble-mean 500-hPa heights (m). The large dot denotes Joaquin's position. (c),(d) As in (a),(b), but for the 12-h forecast.

direction of the 72-h major axis for the 10 most northern and southern members, which is computed in the same manner as the Debby forecast above. During the first $6 \mathrm{~h}$, the northern and southern members are characterized by small, statistically insignificant differences in the ensemble-mean and perturbation steering wind; this corresponds to the time when Joaquin is outside of the sensitive region described above. Between 12 and $36 \mathrm{~h}$, the northern members acquire a $0.4-0.9 \mathrm{~m} \mathrm{~s}^{-1}$ larger southerly component of the perturbation steering wind (statistically significant), as well as a statistically larger southerly ensemble-mean wind that subsequently increases with time. By $60 \mathrm{~h}$, the perturbation steering flow differences are $1.3 \mathrm{~m} \mathrm{~s}^{-1}$, compared to $3.0 \mathrm{~m} \mathrm{~s}^{-1}$ for the ensemble-mean steering flow, with the latter increasing to $6.0 \mathrm{~m} \mathrm{~s}^{-1}$ by $72 \mathrm{~h}$. Similar to the Debby forecast, it appears that the position differences originate from uncertainty in the near-storm steering flow during the first $12 \mathrm{~h}$ of the forecast. This perturbation steering flow causes the TC to move into a region characterized by a different ensemble-mean wind, which subsequently results in large position displacements later in the forecast.

Subsequent forecasts of Hurricane Joaquin's position exhibit decreased ensemble-mean error and standard deviation, likely due to more certainty of Joaquin's position relative to the steering flow axis of contraction. Whereas the forecast initialized at 1200 UTC 30 September still has 10 members making landfall in the southeastern United States within $96 \mathrm{~h}$ and a roughly equal number that are close to the best track (Fig. 4b), the 0000 UTC 1 October initialization time contains five members that have the more western track, while a much larger number of members have a motion more characteristic of the best track northeasterly motion (Fig. 4c). Focusing on the position forecasts valid at 0000 UTC 3 October, the ensemble-mean position error decreases from $430 \mathrm{~km}$ for the forecast initialized 0000 UTC 30 September to $170 \mathrm{~km}$ in the forecast initialized $24 \mathrm{~h}$ later (Fig. 12b). Furthermore, the ensemble standard deviation decreases by over a factor of 2 in between these initialization times. These later forecasts have more certainty on which side of the deformation flow Joaquin will move and hence have less error and uncertainty.

\section{c. Typhoon Lionrock (2016)}

Unlike the previous two cases, there is little correlation between Lionrock's 72-h distance along the major axis (the focus of the subsection) and the distance along the major axis at other lead times during the first $24 \mathrm{~h}$ of the forecast (Fig. 7c). Specifically, the correlation is not 
statistically significant until $36 \mathrm{~h}$ into the forecast, at which point the correlation quickly increases to 0.6 . In turn, it appears that the 72-h position forecast is not as sensitive to the position forecast early in the forecast.

Instead, there appears to be significant correlation between the component of the steering flow in the direction of the major axis and the 72-h position. For this case, the optimal steering flow parameters are the 200$850-\mathrm{hPa}$ layer-average wind with a $333-\mathrm{km}$ TC removal radius (Fig. $8 \mathrm{c}$ ). While the correlation at $0 \mathrm{~h}$ is not statistically significant, by $12 \mathrm{~h}$, the correlation with the steering flow exceeds 0.5 and increases to nearly 1.0 by $60 \mathrm{~h}$, before decreasing thereafter due to the large variability in the steering flow that is a consequence of the over $1000-\mathrm{km}$ difference in position between members (Fig. 7c). The large correlation with the steering flow before $24 \mathrm{~h}$ suggests that the $72-\mathrm{h}$ position forecasts are dependent on the steering flow early in the forecast. Furthermore, variability in the 12 -h column-integrated steering flow in the direction of the major axis ${ }^{2}$ is most sensitive to variability in the $500-\mathrm{hPa}$ wind, with comparatively less sensitivity above and below that (Fig. 9c).

For this case, the 72-h position forecast exhibits large sensitivity to subtle variations in the 0 -h steering flow in between Lionrock and the midlatitude trough to its northwest. Figure 14a indicates that Lionrock's 72-h position forecast has large sensitivity to the 0 -h component of the steering flow along the 72-h major axis of variability to the north of the TC along $30^{\circ} \mathrm{N}$, such that making the wind more southeasterly by one standard deviation is associated with Lionrock being $300 \mathrm{~km}$ to the northwest at $72 \mathrm{~h}$. This region is along the southern end of the deep-layer trough in the steering wind over eastern China and a short-wave ridge to the east of the trough and north of Lionrock. This pattern of sensitivity suggests that Lionrock's 72-h position is sensitive to the southern extent of the midlatitude westerlies, such that shifting this region to the north (which would result in a perturbation southeasterly wind) is associated with a more northwestern 72-h position. Twelve hours later, the 72-h position forecast remains sensitive to the steering flow along the southern end of the trough, but the sensitive region expands in area and includes the immediate region around Lionrock and on the southern edge of the anticyclone to the southeast of Lionrock (Fig. 14b). It is worth pointing out that most of the track sensitivity is associated with the component of the wind normal to the mean steering flow and to the axis of contraction, similar to the previous cases.

\footnotetext{
${ }^{2}$ First time where the steering flow correlation is statistically significant.
}

The evolution of the steering flow near Lionrock appears to be related to uncertainty in two features in the nearby $500-\mathrm{hPa}$ height field that subsequently evolve with time (Fig. 15). At $0 \mathrm{~h}$, the first region of large sensitivity is a west-east negative-positive dipole centered on $29^{\circ} \mathrm{N}, 132^{\circ} \mathrm{E}$, which is just north of Lionrock and brackets a larger-scale trough centered along $130^{\circ} \mathrm{E}$ (Fig. 15a). This pattern of sensitivity indicates that lowering the heights to the west of Lionrock and/or increasing the heights to the east by one standard deviation is associated with Lionrock being $240 \mathrm{~km}$ to the northwest of the ensemble mean position at $72 \mathrm{~h}$. The combination of negative heights to the west and positive heights to the east would imply a southerly perturbation geostrophic wind acting upon Lionrock during subsequent lead times. The second main region of sensitivity is associated with the western side of the trough over eastern China (centered on $45^{\circ} \mathrm{N}, 120^{\circ} \mathrm{E}$ ), such that increasing the heights to the west of the trough, which in turn would imply a more amplified upstream ridge, is associated with a more northwestern position of Lionrock. Twelve hours later, the sensitive regions appear to move with Lionrock and the eastern China trough (Fig. 15c). The sensitivity to the 24-h 500-hPa height field near Lionrock takes on a quadripole pattern, which appears to be a combination of two orthogonal dipoles centered on Lionrock (Fig. 15e). The first negative-positive southwest-northeast dipole appears to reflect the sensitivity to the larger-scale trough originally located at $130^{\circ} \mathrm{E}$, while the second northwest-southeast negative-positive dipole is a reflection of the sensitivity to Lionrock's position at this time. Displacing Lionrock to the northwest at $24 \mathrm{~h}$ will result in the TC being more to the northwest at $72 \mathrm{~h}$ as well (also seen in Fig. 5c). Furthermore, the region of positive sensitivity upstream of the eastern China trough remains within the ridge upstream of the ensemble-mean trough, with a region of negative sensitivity now on the south side of the trough. This region of negative sensitivity indicates that Lionrock will have a more northwest $72-\mathrm{h}$ position if this trough moves farther equatorward at this time. It is likely that this negative sensitivity region is related to the upstream positive sensitivity region since increasing the heights over Mongolia at $0 \mathrm{~h}$ would imply a northerly geostrophic wind over the downstream trough, which in turn would be expected to advect this trough to the south over time (the sensitivity to the $250-\mathrm{hPa} \mathrm{PV}$ is consistent with this hypothesis; not shown).

In addition to the sensitivity associated with the location of various synoptic features, Lionrock's position forecast appears to have a secondary sensitivity to the amplitude of the diabatic outflow. During the first $12 \mathrm{~h}$, 


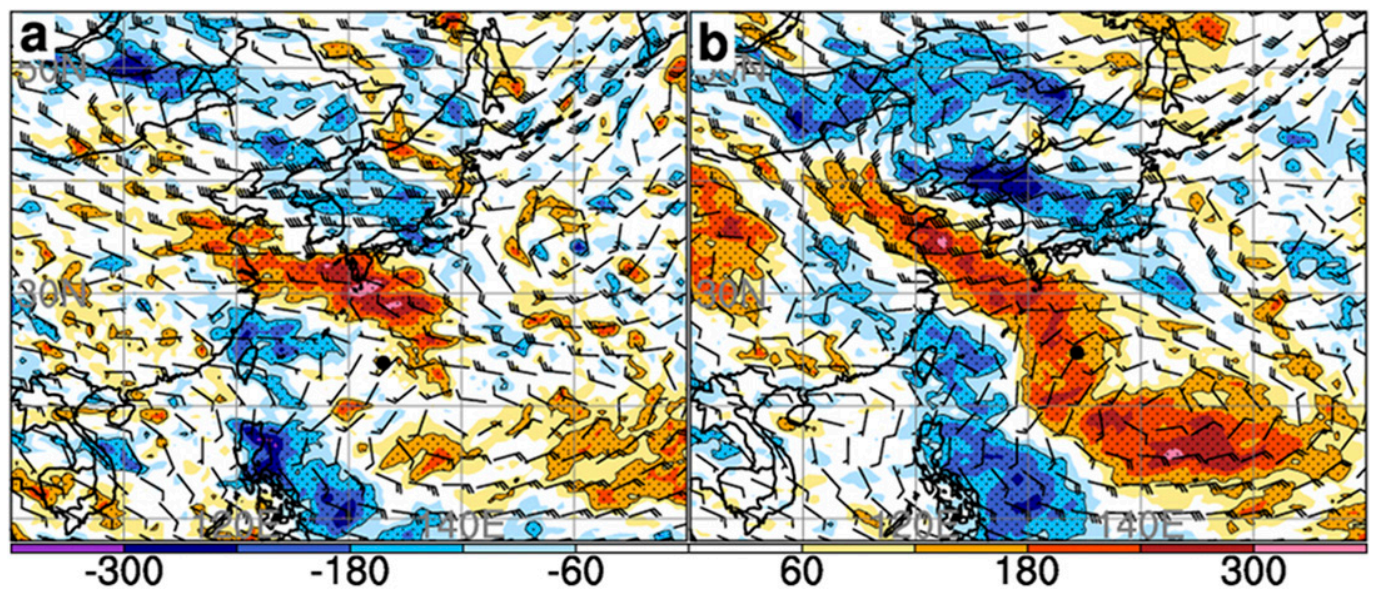

FIG. 14. Sensitivity of Lionrock's 72 -h distance along the major axis to the (a) 0 - and (b) 12-h components of the steering wind in the direction of the 72-h major axis (shading, $\mathrm{km}$ ). Stippled regions indicate where the sensitivity is statistically significant at the $95 \%$ confidence level. The barbs denote the ensemble-mean steering wind. The large dot denotes Lionrock's position.

the sensitivity to the $200-300-\mathrm{hPa}$ divergence (used as a proxy for diabatic outflow) is scattered and smaller in amplitude compared to the 500-hPa height (Figs. 15b,d). By $24 \mathrm{~h}$, there is a region of positive divergence sensitivity collocated with the ensemble-mean divergence over South Korea, which is downstream of the ensemble-mean trough (Fig. 15f). The increased divergence subsequently leads to a more negatively tilted PV anomaly, which in turn would be expected to impart a more southeasterly steering wind on Lionrock (not shown).

Much of the position difference between the 10 most northwest and southeast members is explained by a transition from differences in the perturbation steering wind to differences in the ensemble-mean steering wind (Fig. 11c). By $12 \mathrm{~h}$, the perturbation steering wind in the direction of the 72-h major axis is $0.7 \mathrm{~m} \mathrm{~s}^{-1}$ higher for the northwest members compared to the southeast members. These differences increase to $1.2 \mathrm{~m} \mathrm{~s}^{-1}$ by $48 \mathrm{~h}$, which would yield no more than a $104 \mathrm{~km} \mathrm{day}^{-1}$ position difference. By contrast, the difference in the ensemblemean wind is less than $0.5 \mathrm{~m} \mathrm{~s}^{-1}$ through $36 \mathrm{~h}$, but then increases in an exponential manner thereafter, so that by $60 \mathrm{~h}$, the northwestern members have a $9 \mathrm{~m} \mathrm{~s}^{-1}$ wind along the major axis, while the southeastern members are closer to $0 \mathrm{~m} \mathrm{~s}^{-1}$. At $72 \mathrm{~h}$, the northwestern members have a $22 \mathrm{~m} \mathrm{~s}^{-1}$ ensemble-mean wind, while the southeastern members remain near zero.

Similar to the Debby and Joaquin forecasts, the ensemble-mean error and standard deviation significantly decrease at later initialization times when it becomes clearer on which side of the axis of contraction Lionrock will move. While the 1200 UTC 27 August initialization still contains a large number of members that stall east of Japan (Fig. 6b), nearly all members from the 0000 UTC 28 August initialization replicate the actual northwesterly motion over Japan (Fig. 6c). Moreover, there is a $85 \%$ reduction in the ensemblemean position error and $65 \%$ reduction in the ensemble position standard deviation for 0000 UTC 30 August between the 0000 UTC 27 August and 0000 UTC 28 August initialization time (Fig. 12c).

\section{Summary and conclusions}

This study evaluates the sensitivity of TC position forecasts within the ECMWF ensemble for three cases (Debby, Joaquin, and Lionrock) characterized by large anisotropic, cross-track position variability. In all three cases, the TC is initially located near or along the axis of contraction of a large-scale steering flow characterized by deformation. The relative contribution of uncertainty in the near-storm steering flow versus more remote steering flow uncertainty is evaluated by computing the ensemble-based sensitivity of the position forecast along the axis of greatest position variability to the casespecific steering flow at various lead times.

For all three cases, the largest position forecast sensitivity is mainly tied to variability in the near-storm steering flow. For Debby and Joaquin, differences in the 0-12-h steering flow, on the order of $0.5 \mathrm{~m} \mathrm{~s}^{-1}$, between the ensemble members lead to the TC moving onto either side of the axis of contraction of the deformation wind field, while in Lionrock, the important steering flow differences occur during the first $24 \mathrm{~h}$. As the TC moves onto either side of the axis of contraction, it will experience a different ensemble-mean steering wind, 


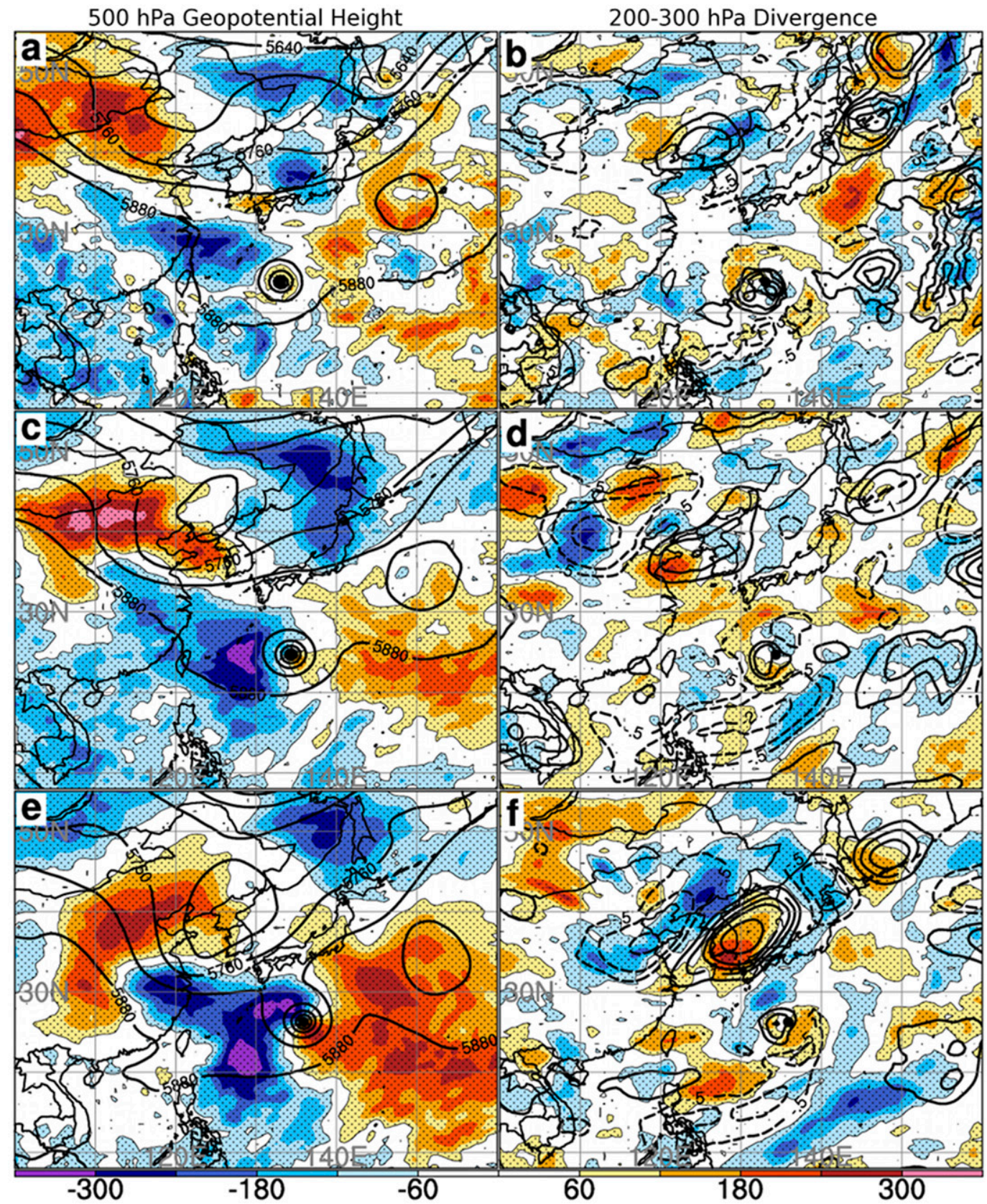

FIG. 15. Sensitivity of Lionrock's 72-h distance along the major axis to the (a) 0-, (c) 12-, and (e) 24-h 500-hPa geopotential height (shading, $\mathrm{km}$ ). Stippled regions indicate where the sensitivity is statistically significant at the $95 \%$ confidence level. The contours denote the ensemble-mean 500-hPa geopotential height. The large dot denotes Lionrock's position. (b),(d),(f) As in (a),(c),(e), but for the 200-300-hPa divergence $\left(10^{5} \mathrm{~s}^{-1}\right)$.

which subsequently leads to the TC accelerating away from the ensemble-mean position, while the differences in the perturbation steering flow remain comparatively smaller through the remainder of the forecast. By contrast, the position forecasts exhibit comparatively less sensitivity to steering flow uncertainty more than $500 \mathrm{~km}$ from the initial TC position. Further support for this paradigm is provided by later forecast lead times, which show large decreases in both ensemble-mean position error and standard deviation as it becomes clearer to which side of the axis of contraction the TC will eventually move. It is worth pointing out that later initialization times have comparable 0-h TC steering wind standard deviations; however, these perturbation steering winds 
are not sufficient to cause the TC to move onto the other side of the axis of contraction.

Although previously documented cases were characterized by different synoptic situations and evolutions, the results presented here are consistent with their conclusions. In particular, these results are similar to those of Grams et al. (2013), who showed that small differences in the position of Typhoon Jangmi relative to the saddle point created by the midlatitude Rossby wave pattern led to large TC position and evolution changes. In contrast to Hurricane Sandy's forecasts, variability in convection and diabatic outflow do not appear to be the primary sensitivity for the position forecasts (e.g., Bassill 2014; Torn et al. 2015); however, the results are similar in that Sandy was found to move onto either side of the axis of contraction depending on the interaction between convection and the steering flow in forecasts initialized 5 days prior to landfall. Furthermore, Hurricane Sandy forecasts initialized at later times also exhibited significant reductions in position error and variability as it became clear that Sandy would move to the west of the axis of contraction. Finally, the results from Joaquin broadly agree with the conclusions of Nystrom et al. (2018), who found that replacing the initial conditions $600-900 \mathrm{~km}$ from Joaquin's center (consistent with the sensitivity region identified here) with another convection-resolving analysis yielded improved track forecasts using the Weather Research and Forecasting (WRF) Model. This result is intriguing in that a similar answer was found despite using different models and initial condition sources.

These results have some important implications for how to account for future TCs that occur in similar steering flows. One potential way to reduce the uncertainty in these forecasts would be to sample the steering flow around these TCs either via aircraft data [e.g., the NOAA Gulfstream IV aircraft (G-IV); DOTSTAR, Wu et al. (2005)], which might be difficult if the TC is far from land, or via alternative methods, such as rapid-scan satellite images, which can provide a large number of vector winds, though perhaps not at the level of interest (i.e., $500 \mathrm{hPa}$ ). Moreover, it appears that more remote observations from the TC would have limited value given that these cases exhibit minimal sensitivity to the evolution of the steering flow. The critical aspect here is to sample the steering flow with observations as early as possible in the TC lifetime when the position forecasts will be most sensitive to subtle differences in the steering flow. Over time, the forecasts become more confident as it becomes clear to which side of the axis of contraction the TC will move. Finally, it is clear that TCs in deformation steering flows are inherently difficult to predict given the nature of the wind field, which motivates using ensemble prediction systems, rather than deterministic forecasting, which may have large errors if the forecast moves toward the wrong side of the axis of contraction. Moreover, TC position forecasts might be most sensitive to cases when the perturbation steering flow can cause the TC to move into different ensemble-mean steering winds, which are often larger than the ensemble perturbation wind. Future work will likely pursue computing the relative contribution of perturbation steering wind and ensemble-mean steering wind gradients on TC position variability over a larger set of cases. Furthermore, it is also worthwhile to investigate the frequency of TCs in deformation steering flows and the extent to which position forecasts in these situations are less predictable than a typical position forecast.

Acknowledgments. This work benefited from discussions with Nick Bassill. The authors are grateful to ECMWF for providing access to past ECMWF forecasts through the TIGGE portal. Three anonymous reviewers provided helpful feedback on an earlier version of this manuscript. This research is supported by NOAA Awards NA14NWS4680027 and NA16NWS4680025. The National Center for Atmospheric Research is supported by the National Science Foundation.

\section{REFERENCES}

Aberson, S. D., and M. DeMaria, 1994: Verification of a nested barotropic hurricane track forecast model (VICBAR). Mon. Wea. Rev., 122, 2804-2815, https://doi.org/10.1175/15200493(1994)122<2804:VOANBH>2.0.CO;2.

Ancell, B., and G. J. Hakim, 2007: Comparing adjoint and ensemble sensitivity analysis with applications to observation targeting. Mon. Wea. Rev., 135, 4117-4134, https://doi.org/ 10.1175/2007MWR1904.1.

Anwender, D., P. A. Harr, and S. C. Jones, 2008: Predictability associated with the downstream impacts of the extratropical transition of tropical cyclones: Case studies. Mon. Wea. Rev., 136, 3226-3247, https://doi.org/10.1175/2008MWR2249.1.

Archambault, H. M., D. Keyser, L. F. Bosart, and J. M. Cordeira, 2013: A climatological analysis of the extratropical flow response to recurving western North Pacific tropical cyclones. Mon. Wea. Rev., 141, 2325-2346, https://doi.org/10.1175/MWR-D-12-00257.1.

Bassill, N. P., 2014: Accuracy of early GFS and ECMWF Sandy (2012) track forecasts: Evidence for a dependence on cumulus parameterization. Geophys. Res. Lett., 41, 3274-3281, https:// doi.org/10.1002/2014GL059839.

Berg, R., 2016: Hurricane Joaquin (AL112015) 28 September7 October 2015. National Hurricane Center Tropical Cyclone Rep., 36 pp., https://www.nhc.noaa.gov/data/tcr/ AL112015_Joaquin.pdf.

Blake, E. S., T. B. Kimberlain, R. J. Berg, J. P. Cangialosi, and J. L. Beven, 2013: Hurricane Sandy (AL182012) 22-29 October 2013. National Hurricane Center Tropical Cyclone Rep., 157 pp., http://www.nhc.noaa.gov/data/tcr/AL182012_Sandy.pdf.

Bougeault, P., and Coauthors, 2010: The THORPEX Interactive Grand Global Ensemble. Bull. Amer. Meteor. Soc., 91, 10591072, https://doi.org/10.1175/2010BAMS2853.1. 
Carr, L. E., and R. L. Elsberry, 2000: Dynamical tropical cyclone track forecast errors. Part I: Tropical region error sources. Wea. Forecasting, 15, 641-661, https://doi.org/ 10.1175/1520-0434(2000)015<0641:DTCTFE > 2.0.CO;2.

Chan, J. C. L., and W. M. Gray, 1982: Tropical cyclone movement and surrounding flow relationships. Mon. Wea. Rev., 110, 1354-1374, https://doi.org/10.1175/1520-0493(1982)110<1354: TCMASF $>2.0 . \mathrm{CO} ; 2$.

Chen, J.-H., M. S. Peng, C. A. Reynolds, and C.-C. Wu, 2009: Interpretation of tropical cyclone forecast sensitivity from the singular vector perspective. J. Atmos. Sci., 66, 3383-3400, https://doi.org/10.1175/2009JAS3063.1.

Dong, K., and C. J. Neumann, 1986: The relationship between tropical cyclone motion and environmental geostrophic flows. Mon. Wea. Rev., 114, 115-122, https://doi.org/10.1175/ 1520-0493(1986)114<0115:TRBTCM>2.0.CO;2.

Galarneau, T. J., and C. A. Davis, 2013: Diagnosing forecast errors in tropical cyclone motion. Mon. Wea. Rev., 141, 405-430, https://doi.org/10.1175/MWR-D-12-00071.1.

George, J. E., and W. M. Gray, 1976: Tropical cyclone motion and surrounding parameter relationships. J. Appl. Meteor., 15, 1252-1264, https://doi.org/10.1175/1520-0450(1976)015<1252: TCMASP $>2.0 . C O ; 2$.

Gombos, D., R. N. Hoffman, and J. A. Hansen, 2012: Ensemble statistics for diagnosing dynamics: Tropical cyclone track forecast sensitivities revealed by ensemble regression. Mon. Wea. Rev., 140, 2647-2669, https://doi.org/10.1175/ MWR-D-11-00002.1.

Grams, C. M., S. C. Jones, C. A. Davis, P. A. Harr, and M. Weissmann, 2013: The impact of Typhoon Jangmi (2008) on the midlatitude flow. Part II: Downstream evolution. Quart. J. Roy. Meteor. Soc., 139, 2165-2180, https://doi.org/10.1002/qj.2119.

Hamill, T. M., J. S. Whitaker, M. Fiorino, and S. J. Benjamin, 2011: Global ensemble predictions of 2009's tropical cyclones initialized with an ensemble Kalman filter. Mon. Wea. Rev., 139, 668-688, https://doi.org/10.1175/2010MWR3456.1.

— G. T. Bates, J. S. Whitaker, D. R. Murray, M. Fiorino, T. J. Galarneau, Y. Zhu, and W. Lapenta, 2013: NOAA's secondgeneration global medium-range ensemble reforecast dataset. Bull. Amer. Meteor. Soc., 94, 1553-1565, https://doi.org/ 10.1175/BAMS-D-12-00014.1.

Harr, P. A., D. Anwender, and S. C. Jones, 2008: Predictability associated with the downstream impacts of the extratropical transition of tropical cyclones: Methodology and a case study of Typhoon Nabi (2005). Mon. Wea. Rev., 136, 3205-3225, https://doi.org/10.1175/2008MWR2248.1.

Henderson, J. M., G. M. Lackmann, and J. R. Gyakum, 1999: An analysis of Hurricane Opal's forecast track errors using quasigeostrophic potential vorticity inversion. Mon. Wea. Rev., 127, 292-307, https://doi.org/10.1175/1520-0493(1999)127<0292: AAOHOS $>2.0 . \mathrm{CO} ; 2$.

Holland, G. J., 1983: Tropical cyclone motion: Environmental interaction plus beta effect. J. Atmos. Sci., 40, 328-342, https:// doi.org/10.1175/1520-0469(1983)040<0328:TCMEIP > 2.0.CO;2.

Hoover, B. T., C. S. Velden, and S. J. Majumdar, 2013: Physical mechanisms underlying selected adaptive sampling techniques for tropical cyclones. Mon. Wea. Rev., 141, 4008-4027, https://doi.org/10.1175/MWR-D-12-00269.1.

Ido, K., and C.-C. Wu, 2013: Typhoon-position-oriented sensitivity analysis. Part I: Theory and verification. J. Atmos. Sci., 70, 2525-2546, https://doi.org/10.1175/JAS-D-12-0301.1.

Kimberlain, T. B., 2013: Tropical Storm Debby (AL042012) 23-27 June 2012. National Hurricane Center Tropical
Cyclone Rep., 51 pp., http://www.nhc.noaa.gov/data/tcr/ AL042012_Debby.pdf.

Komaromi, W. A., S. J. Majumdar, and E. D. Rappin, 2011: Diagnosing initial condition sensitivity of Typhoon Sinlaku (2008) and Hurricane Ike (2008). Mon. Wea. Rev., 139, 32243242, https://doi.org/10.1175/MWR-D-10-05018.1.

Landsea, C. W., and J. L. Franklin, 2013: Atlantic hurricane database uncertainty and presentation of a new database format. Mon. Wea. Rev., 141, 3576-3592, https://doi.org/10.1175/MWR-D-12-00254.1. , and J. P. Cangialosi, 2018: Have we reached the limits of predictability for tropical cyclone track forecasting? Bull. Amer. Meteor. Soc., https://doi.org/10.1175/BAMS-D-17-0136.1, in press.

Majumdar, S. J., S. D. Aberson, C. H. Bishop, R. Buizza, M. S. Peng, and C. A. Reynolds, 2006: A comparison of adaptive observing guidance for Atlantic tropical cyclones. Mon. Wea. Rev., 134, 2354-2372, https://doi.org/10.1175/MWR3193.1.

Munsell, E. B., and F. Zhang, 2014: Prediction and uncertainty of Hurricane Sandy (2012) explored through a real-time cloudpermitting ensemble analysis and forecast system assimilating airborne Doppler radar observations. J. Adv. Model. Earth Syst., 6, 38-58, https://doi.org/10.1002/2013MS000297.

Nystrom, R. G., F. Zhang, E. B. Munsell, S. A. Braun, J. A. Sippel, Y. Weng, and K. Emanuel, 2018: Predictability and dynamics of Hurricane Joaquin (2015) explored through convectionpermitting ensemble sensitivity experiments. J. Atmos. Sci., 75, 401-424, https://doi.org/10.1175/JAS-D-17-0137.1.

Peng, M. S., and C. A. Reynolds, 2006: Sensitivity of tropical cyclone forecasts as revealed by singular vectors. J. Atmos. Sci., 63, 2508-2528, https://doi.org/10.1175/JAS3777.1.

Podlaha, A., S. Bowen, C. Darbinyan, and M. Lorinc, 2016: Global catastrophe recap, October 2016. Aon Benfield Tech. Rep., $18 \mathrm{pp}$. , http://thoughtleadership.aonbenfield.com/Documents/ 20161109-ab-analytics-if-october-global-recap.pdf.

Rappaport, E. N., and Coauthors, 2009: Advances and challenges at the National Hurricane Center. Wea. Forecasting, 24, 395419, https://doi.org/10.1175/2008WAF2222128.1.

Riemer, M., and S. C. Jones, 2014: Interaction of a tropical cyclone with a high-amplitude, midlatitude wave pattern: Waviness analysis, trough deformation and track bifurcation. Quart. J. Roy. Meteor. Soc., 140, 1362-1376, https://doi.org/10.1002/qj.2221.

Torn, R. D., and G. J. Hakim, 2008: Ensemble-based sensitivity analysis. Mon. Wea. Rev., 136, 663-677, https://doi.org/10.1175/ 2007MWR2132.1.

__ and C. Snyder, 2012: Uncertainty of tropical cyclone besttrack information. Wea. Forecasting, 27, 715-729, https:// doi.org/10.1175/WAF-D-11-00085.1.

— J. S. Whitaker, P. Pegion, T. M. Hamill, and G. J. Hakim, 2015: Diagnosis of the source of GFS medium-range track errors in Hurricane Sandy (2012). Mon. Wea. Rev., 143, 132152, https://doi.org/10.1175/MWR-D-14-00086.1.

Velden, C., and L. M. Leslie, 1991: The basic relationship between tropical cyclone intensity and the depth of the environmental steering layer in the Australian region. Wea. Forecasting, 6 , 244-253, https://doi.org/10.1175/1520-0434(1991)006<0244: TBRBTC $>2.0 . \mathrm{CO} ; 2$.

Wu, C. C., and K. A. Emanuel, 1995a: Potential vorticity diagnostics of hurricane movement. Part I: A case study of Hurricane Bob (1991). Mon. Wea. Rev., 123, 69-92, https://doi.org/10.1175/ 1520-0493(1995)123<0069:PVDOHM >2.0.CO;2. , and _ 1995b: Potential vorticity diagnostics of hurricane movement. Part II: Tropical Storm Ana (1991) and Hurricane Andrew (1992). Mon. Wea. Rev., 123, 93-109, https://doi.org/ 10.1175/1520-0493(1995)123<0093:PVDOHM>2.0.CO;2. 
- T.-S. Huang, and K.-H. Chou, 2004: Potential vorticity diagnosis of the key factors affection the motion of Typhoon Sinlaku (2002). Mon. Wea. Rev., 132, 2084-2093, https://doi.org/ 10.1175/1520-0493(2004)132<2084:PVDOTK>2.0.CO;2.

_, and Coauthors, 2005: Dropwindsonde Observations for Typhoon Surveillance near the Taiwan Region (DOTSTAR): An overview. Bull. Amer. Meteor. Soc., 86, 787-790, https:// doi.org/10.1175/BAMS-86-6-787.

—, J.-H. Chen, P.-H. Lin, and K.-H. Chou, 2007: Targeted observations of tropical cyclone movement based on the adjoint-derived sensitivity steering vector. J. Atmos. Sci., 64, 2611-2626, https://doi.org/10.1175/JAS3974.1.

and Coauthors, 2009: Intercomparison of targeted observation guidance for tropical cyclones in the northwestern Pacific. Mon. Wea. Rev., 137, 2471-2492, https://doi.org/10.1175/ 2009MWR2762.1.

Yamaguchi, M., J. Ishida, H. Sato, and M. Nakagawa, 2017: WGNE intercomparison of tropical cyclone forecasts by operational NWP models: A quarter century and beyond. Bull. Amer. Meteor. Soc., 98, 2337-2349, https://doi.org/10.1175/BAMS-D-16-0133.1. 